\title{
Sonic hedgehog signaling regulates actin cytoskeleton via Tiam1-Rac1 cascade during spine formation.
}

\section{$\operatorname{AUTHOR}(S)$ :}

Sasaki, Nobunari; Kurisu, Junko; Kengaku, Mineko

\section{CITATION:}

Sasaki, Nobunari ...[et al]. Sonic hedgehog signaling regulates actin cytoskeleton via

Tiam1-Rac1 cascade during spine formation.. Molecular and cellular neurosciences 2010,

45(4): 335-344

ISSUE DATE:

2010-12

URL:

http://hdl.handle.net/2433/131839

\section{RIGHT:}

(C) 2010 Elsevier Inc:; This is not the published version. Please cite only the published version.; この論文は出版社版でありません。引用の際に は出版社版をご確認ご利用ください。 
Sonic hedgehog signaling regulates actin cytoskeleton via Tiam1-Rac1 cascade during spine formation

Nobunari Sasaki $^{a, b}$, Junko Kurisu ${ }^{\text {a, b }}$ and Mineko Kengaku ${ }^{\text {a, b, * }}$

${ }^{a}$ Laboratory for Neural Cell Polarity, RIKEN Brain Science Institute, 2-1 Hirosawa, Wako, Saitama 351-0198, Japan.

${ }^{\mathrm{b}}$ Institute for Integrated Cell-Material Sciences, Kyoto University, Yoshida-honmachi, Kyoto 606-8501, Japan

*Correspondence: kengaku@icems.kyoto-u.ac.jp

Phone; 81-75-753-9832, Fax; 81-75-753-9820

Running title: Shh signaling regulates actin cytoskeleton

Keywords: Sonic hedgehog, Smoothened, Tiam1, dendritic spine, actin 


\section{Abstract}

The sonic hedgehog (Shh) pathway has essential roles in several processes during development of the vertebrate central nervous system (CNS). Here, we report that Shh regulates dendritic spine formation in hippocampal pyramidal neurons via a novel pathway that directly regulates the actin cytoskeleton. Shh signaling molecules Patched (Ptc) and Smoothened (Smo) are expressed in several types of postmitotic neurons, including cerebellar Purkinje cells and hippocampal pyramidal neurons. Knockdown of Smo induces dendritic spine formation in cultured hippocampal neurons independently of Gli-mediated transcriptional activity. Smo interacts with Tiam1, a guanine nucleotide exchange factor for Rac1, via its cytoplasmic C-terminal region. Inhibition of Tiam1 or Rac1 activity suppresses spine induction by Smo knockdown. Shh induces remodeling of the actin cytoskeleton independently of transcriptional activation in mouse embryonic fibroblasts. These findings demonstrate a novel Shh pathway that regulates the actin cytoskeleton via Tiam1-Rac1 activation. 


\section{Inttroduction}

Sonic hedgehog (Shh) has essential roles during morphogenesis in vertebrates and invertebrates.

Shh is secreted by organizer cells and regulates various biological processes via transcriptional regulation in target cells (McMahon et al., 2003; Varjosalo and Taipale, 2008). In the absence of Shh, its receptor Patched1 (Ptc) inhibits the seven-pass transmembrane protein Smoothened (Smo) and subsequent intracellular signaling. Upon binding of Shh, Ptc releases Smo which then mediates Shh signaling by activating intracellular molecules including the Gli family transcription factors (Huangfu and Anderson, 2006; Ruiz i Altaba et al., 2002). Loss of Shh signaling activity causes malformations in various tissues, whereas aberrant activation of Shh pathway is associated with human cancers including basal cell carcinoma and medulloblastoma (Beachy et al., 2004; Varjosalo and Taipale, 2008). During development of the vertebrate central nervous system (CNS), Shh signaling is required for ventral patterning of the neural tube and proliferation and differentiation of neural progenitors (Fuccillo et al., 2006). Recent studies have also implicated Shh signaling in the guidance of growing axons (Hammond et al., 2009; Yam et al., 2009).

Dendritic spines are small actin-rich protrusions on the dendritic surface that form postsynaptic contacts receiving excitatory inputs. Spines undergo dynamic changes in size, number and shape during neuronal development and by extracellular stimuli such as neurotrophic factors and neuronal activity (Calabrese et al., 2006; Ethell and Pasquale, 2005). The formation and remodeling of spines are mainly regulated by modification of the actin cytoskeleton. Rho family small GTPases are key 
regulators of actin cytoskeleton and thought to be essential for spine formation (Cingolani and Goda, 2008; Schubert and Dotti, 2007). Rho GTPases are molecular switches converting their conformation between an inactive GDP bound form and an active GTP bound form. The activities of Rho GTPases are strictly regulated by guanine nucleotide exchange factors (GEFs), GTPase-activating proteins (GAPs) and guanine nucleotide dissociation inhibitors (GDIs) (Jaffe and Hall, 2005). Tiam1 (T-lymphoma invasion and metastasis) is a Rac1-specific GEF that activates Rac1 by catalyzing the exchange of GDP for GTP. It has been shown that Tiam1 is localized to dendritic spines by interacting with PAR3 and that the Tiam1-Rac1 cascade regulates spine formation downstream of EphB2 and NMDA receptors in hippocampal neurons (Tolias et al., 2005; Tolias et al., 2007; Zhang and Macara, 2006).

Here, we report that Smo interacts with Tiam1 and regulates dendritic spine formation. Shh signaling induces the reorganization of the actin cytoskeleton via activation of the Tiam1-Rac1 cascade independently of transcriptional activity. We propose that a novel Shh pathway regulates the remodeling of the actin cytoskeleton through Tiam1-Rac1 and regulates the actin-dependent remodeling required for dendritic spine formation. 


\section{Results}

\section{Shh signaling is implicated in dendritic spine formation}

In an attempt to explore additional roles of the Shh pathway during CNS development, expression patterns of hedgehog signaling molecules were analyzed by in situ hybridization in early postnatal mouse brain. We found that Shh was co-expressed with Ptc and Smo in cerebellar Purkinje neurons and hippocampal pyramidal neurons in postnatal day 14 (P14) mice (Fig. 1). It is thus suggested that Shh signaling might function in postmitotic CNS neurons.

To analyze the function of Shh signaling in postmitotic neurons, we examined the effects of Shh on cultured hippocampal neurons. At 16 days in vitro (16 DIV), neurons have abundant mature spines with a thin neck and large head as well as longer and thinner headless processes called filopodia (Fig. 2A-a). Under normal conditions, neurons initially grow thin headless filopodia, which are subsequently modified into spines along with the maturation of synapses (Ethell and Pasquale, 2005; Matsuno et al., 2006). In our culture, the number of spines continuously increased until 26 DIV, while that of filopodia plateaued after 16 DIV (Supplemental Figs. S1A, B). We found that recombinant Shh peptide treatment significantly increased the density of spines compared to untreated control neurons (Figs. 2A-a, b, B). Conversely, neurons treated with cyclopamine, which binds to Smo and inhibits its activity to transduce Shh signaling (Chen et al., 2002a), exhibited lower density of spines and higher density of dendritic filopodia (Figs. 2A-c, d, B and S1C). We also examined the effect of another Smo antagonist SANT-1 which has been shown to work 
upstream of cyclopamine and interfere with the maturation of Smo (Chen et al., 2002b; Rohatgi et al., 2009). Unexpectedly, SANT-1 treatment had an opposing effect to cyclopamine and markedly increased the density of spines (Figs. 2A-e, B). To further investigate the role of Smo in spine formation, we employed a microRNA based RNA interference (miR RNAi) system for silencing endogenous expression of Smo in neurons. Two distinct Smo miR RNAi vectors (SmoRNAi-1 and -2) markedly reduced Smo expression and inhibited the activation of a Gli-luciferase reporter by Shh (Figs. S2A-C). Hippocampal neurons transfected with SmoRNAi-1 or SmoRNAi-2 showed a marked increase in the density of mature spines along the dendrites at 16 DIV compared to neurons transfected with a negative control vector (Figs. 2C-a, b, D and S2D). Overexpression of a RNAi-resistant Smo mutant (Smo-R), a rescue construct with point mutations in the SmoRNAi-1-targeting region without changes in amino acid sequences, significantly rescued the effect of SmoRNAi-1 (Figs. 2C-c, D). Thus, the increase in spine formation was caused by the depletion of Smo and not by nonspecific targeting of the Smo RNAi.

The apparent contradiction between the effects of Smo depletion and antagonists prompted us to examine if Shh-induced transcriptional activity is involved in spine formation. Gli proteins (Gli1, 2 and 3) are a family of transcription factors regulated by the Shh signaling pathway downstream of Smo. We generated a miR RNAi vector for Gli2 (Gli2RNAi), a primary activator for the Shh pathway (Bai et al., 2002; Fuccillo et al., 2006). Luciferase reporter assays confirmed significant suppression of promoter activity in cells expressing the Gli2RNAi (Fig. S2B). We also observed that Gli2RNAi reduced Gli2 expression in hippocampal neurons (Fig. S2E). In spite that Gli2RNAi 
suppressed the Shh transcriptional pathway, depletion of Gli2 did not change the number of spines

(Figs. 2C-d, D). These results suggest that the changes in spine formation by Smo depletion and Smo antagonists are independent of Gli-mediated transcriptional activity. The effects of drug treatment and RNAi experiments are summarized in Supplemental Table S1.

\section{Smo interacts with Tiam1 via its intracellular C-terminal region}

The above results imply a novel mechanism through which Shh treatment and Smo depletion induce spine formation. In an attempt to identify unknown downstream signaling components in this pathway, we searched for molecules that bound to the intracellular region of Smo. The intracellular C-terminal region of Smo $(\mathrm{SmoC})$ has crucial roles in the activation of the Shh signaling pathway and receptor trafficking (Kovacs et al., 2008; Meloni et al., 2006; Zhao et al., 2007). We therefore carried out a mass spectrometry analysis to identify molecules that interact with SmoC. Purified GST-fused SmoC protein was used for pull down assays from P14 mouse brain extracts. Co-precipitated proteins were separated by SDS-PAGE and were then analyzed by mass spectrometry. Several proteins that have not been previously implicated in Shh signaling were identified (Fig. 3A). Among these proteins, we focused on Tiam1 and Intersectin-1, respective GEFs for Rho family small GTPases Rac1 and Cdc42, since the morphology and number of spines are known to be regulated by Rho family small GTPases (Cingolani and Goda, 2008; Schubert and Dotti, 2007). Immunoblot analysis revealed that SmoC strongly interacted with Tiam1 and more weakly with intersectin-1 (Fig. 3B). Little or no binding was detected with beta-PIX, another Rac1 
GEF implicated in spine formation (Saneyoshi et al., 2008).

We confirmed Tiam1 expression in hippocampal pyramidal neurons in P14 mouse brain (Fig. 3C-a-f). Tiam1 expression was high in the cell bodies and had a lower punctate expression in the apical dendrites of pyramidal neurons (Fig.3C-g-i).

Using GST-pull down assays, we further examined the region of Tiam1 that was responsible for binding to SmoC with a panel of truncated Tiam1 constructs. We identified the medial domain of Tiam1 (TiamC) containing a pleckstrin homology (PH) domain and a Ras-binding domain (RBD) to be essential for SmoC binding (Figs. 4A, B). TiamC also contains the site required for plasma membrane targeting and Racl activation (Stam et al., 1997). Reciprocal experiments demonstrated that the central part of SmoC including the poly-basic $(\mathrm{PB})$ region $(\mathrm{SmoCFb})$ was sufficient for interacting with Tiam1 (Figs. 4A, C). These results illustrate that Smo specifically interacts with the Rac1 GEF Tiam1 via its intracellular C-terminal region.

\section{Shh induces the reorganization of the actin cytoskeleton in non-neuronal cells}

We next addressed if Shh signaling regulated Tiam1 activity. NIH3T3 cells has been shown to be activated in response to Shh stimuli (Taipale et al., 2000). Further, the Tiam1-Rac1 pathway is involved in the regulation of the actin cytoskeleton in these cells (Buchanan et al., 2000; Michiels et al., 1995). Therefore, we treated NIH3T3 cells with the recombinant Shh peptide and analyzed changes in the actin cytoskeleton. Addition of Shh induced membrane ruffles, suggesting that Shh stimulated the remodeling of the actin cytoskeleton in non-neuronal cells (Figs. 5A-a, b, e, f, B). 
NIH3T3 cells pre-treated with the transcription inhibitor actinomycin D exhibited membrane ruffles comparable to the untreated control cells, indicating that Shh induced membrane ruffles independently of transcriptional activation (Figs. 5A-c, d, g, h, B). In contrast, overexpression of a dominant-negative form of Tiam1 (Tiam1 DN) completely suppressed the induction of membrane ruffles by Shh. We also found that Shh induced the accumulation of Tiam1 at the edge of membrane ruffles (Figs 5B-D). These results suggest that Shh signaling induces actin remodeling via Tiam1 independently of the transcriptional Shh pathway.

\section{Shh induces spine formation through the Tiam1-Rac1 pathway}

Finally, we confirmed whether Shh regulates spine formation via Tiam1-Rac1 activation. It has been shown that Tiam1 activity is inhibited by overexpression of the medial segment of Tiam1 containing the PH and RBD domains, which is identical to TiamC (Kawauchi et al., 2003; Tolias et al., 2007)). We thus utilized TiamC as a dominant negative form of Tiam1 (Tiam1 DN) (Fig. 6A). Inhibition of Tiam1-Rac1 pathway in cultured pyramidal neurons by overexpression of Tiam1 DN or treatment with Rac1 inhibitor severely decreased spine formation as previously reported (Figs. 6B-a-c, C) (Tolias et al., 2007; Zhang and Macara, 2006). Tiam1 DN and Rac1 inhibitor markedly suppressed the enhancement of spine formation by Smo RNAi, supporting a direct link between Smo and the Tiam1-Rac1 pathway (Figs. 6B-f-h, C). In contrast, overexpression of the RNAi-resistant Smo mutant lacking the Tiam1-binding domain ( $\operatorname{SmoR} \Delta \mathrm{CFb})$ failed to rescue the enhancement of spine formation by Smo RNAi (Figs. 6A, B-i, C). Conversely, overexpression of 
the Smo CF fragment containing the Tiam1-binding domain, which failed to affect the transcriptional pathway, significantly reduced the number of spines (Figs. 6A, B-d, S2B and Table. S1). These results support that Tiam1-binding of Smo inhibits spine formation. In contrast, a constitutively active form of Smo (SmoA1) had little or no effect on spine formation in spite of its strong transcriptional activity (Figs. 6A, B-e, S2B and Table S1). Together, these results suggest that Shh regulates spine formation via the Tiam1-Rac1 pathway independently of transcriptional activation. 


\section{Discussion}

Here we have shown that Shh signaling directly regulates the actin cytoskeleton via Tiam1-Rac1 independently of the transcriptional pathway. Induction of spine formation by Shh treatment was not mediated by Gli2-dependent transcriptional activation. Unlike this Gli-dependent pathway, which is inhibited by Smo depletion, spine induction was not blocked but rather enhanced by Smo RNAi or by treatment with a Smo inhibitor SANT-1. The intracellular C-terminal tail of Smo interacted with Tiam1 and overexpression of the Tiam1-binding domain of Smo inhibited spine formation. Moreover, Shh induced translocation of Tiam1 to membrane ruffles in NIH3T3 cells, suggesting that Smo affects the localization of Tiam1 upon activation by Shh.

Taken together, we propose a model of a novel Shh signaling pathway through Tiam1-Rac1 to regulate actin remodeling (Fig. 7): In this model, Smo binds to and sequesters Tiam1 in its inactive state in the absence of Shh or with the inhibitor cyclopamine (I). In the presence of Shh, Smo is released from Ptc inhibition and mediates Gli-dependent transcriptional activity. Upon activation, Smo releases Tiam1, which can then translocate to the plasma membrane and induce actin reorganization via Rac1 (II). Depletion of Smo by RNAi is thought to result in the accumulation of free Tiam1 and subsequently increased Rac1 activation. Treatment with SANT-1 would also deplete Smo and relieve Tiam1 from its inhibitory action, as SANT-1 is thought to interfere with glycosylation of Smo prior to its transport from the Golgi apparatus (0) (Rohatgi et al., 2009). In contrast, cyclopamine likely acts downstream and induce an inactive conformation of Smo (Rohatgi 
et al., 2009; Wilson et al., 2009) that remains bound to and inhibits Tiam1.

The mechanism of activation of the distinct signaling pathway remains unclear. It has been shown that Smo binds to $\beta$-arrestin via its $\mathrm{C}$-terminal tail followed by translocation to the primary cilium that is required for activation of the transcriptional pathway upon Shh stimuli (Kovacs et al., 2008). However, we show that while overexpression of SmoCF significantly inhibited spine formation, it did not affect Gli-mediated transcriptional activity. It has been indicated that ß-arrestin-binding and activation of the transcriptional pathway are triggered by phosphorylation of the Smo C-terminal tail by GRK2 in response to Shh (Meloni et al., 2006). Thus in our study, overexpressed SmoCF might be in its unphosphorylated state, which does not bind to ß-arrestin nor affect transcriptional activity. It may also be that $\beta$-arrestin-binding and subsequent transcriptional activation involve other domains in the Smo C-terminal tail rather than the domain responsible for Tiam1-binding. Future studies will address the precise domains of the Smo C-terminal tail regulating differential Shh pathways.

In our model, Shh signaling has a permissive role in the Tiam1-Rac1 pathway. We found that Shh induced actin remodeling in NIH3T3 fibroblasts with a slower time course than other stimuli like PDGF, which is directly involved in Tiam1 activation (Anton et al., 2003). Consistent with our observation, translocation of Smo into primary cilium has been shown to occur in the similar time frame after Shh treatment (Rohatgi et al., 2007). The slow activation of Smo might be prerequisite but not sufficient for activation of the Tiam1-Rac1 pathway. We surmise that Smo releases Tiam1 in the presence of Shh and a free Tiam1 pool may accumulate in the cytosol, which would then be 
recruited to the plasma membrane by other upstream signals and activate Rac1.

We observed that the number of filopodia plateaued, while spines were consistently increased after 16 DIV in our culture conditions (Fig. S1B). De novo formation of filopodia is thus likely to continue after $16 \mathrm{DIV}$, as filopodia are transformed into dendritic spines after maturation. Based on this, a significant increase in filopodia at the expense of spines after the addition of cyclopamine suggests that Shh signaling pathway would affect the filopodia-to-spine maturation but not de novo formation of filopodia.

In hippocampal neurons and cerebellar Purkinje cells, the ligand and downstream components of Shh signaling are expressed in the same neurons, implying that Shh might regulate spine formation in an autocrine or paracrine manner. This raises the intriguing question of how Shh can regulate spine formation in a specific spatiotemporal manner. Secretion of Shh might be regulated by other extracellular signals at specific locations and times. Interestingly, secretion of Shh has been shown to be triggered by histamine (Zavros et al., 2008), which has also been reported to induce $\mathrm{Ca}^{2+}$ influx in endothelial cells (Rotrosen and Gallin, 1986). This may suggest that activity-dependent $\mathrm{Ca}^{2+}$ influx may be involved in Shh secretion in neurons.

Recent studies have suggested that Shh functions as a chemoattractant independently of its transcriptional activity. Shh has been implicated in growth cone guidance of commissural neurons through the activation of Src-family kinases. It has also been shown that Smo-activation by Shh induces migration of mouse mesenchymal fibroblasts through the metabolism of arachidonic acid (Bijlsma et al., 2007; Yam et al., 2009). Since Src-family kinases and arachidonate metabolites are 
also involved in synaptic function, this suggests a possible link between Smo-Tiam1 pathway and these molecules in spine formation (Feinmark et al., 2003; Salter and Kalia, 2004). Additional studies are needed to delineate the detailed pathways and physiological roles of Shh in dendritic spine formation.

Our identification of a novel Shh signaling pathway in the regulation of the actin cytoskeleton sheds light on new aspects of Shh function. The extent to which this novel signaling cascade is involved in other Shh functions during development should be clarified by future studies. 


\section{Experimental methods}

\section{Antibodies and reagents}

The following antibodies and reagents were used: rabbit polyclonal antibodies against HA-tag, Tiam1 (Santa Cruz Biotechnology), GFP (Molecular Probes), beta PIX (Chemicon); goat polyclonal anti-HA-tag antibody (Abcam); mouse monoclonal antibodies against Calbindin-D-28K (Sigma), Intersectin (BD Biosciences); rhodamine-phalloidin (Molecular Probes), Alexa488 or Alexa568-conjugated anti-rabbit, anti-mouse, or anti-goat IgG (Molecular Probes); horseradish peroxidase (HRP)-conjugated anti-rabbit and anti-mouse IgG (Chemicon); recombinant mouse Shh amino-terminal peptide (R\&D Systems), Cyclopamine (Toronto Research Chemicals), SANT-1 and Rac1 inhibitor NSC23766 (Calbiochem).

\section{Plasmids}

For expression in cultured cells, Venus (a gift of A. Miyawaki) was subcloned into pCAGGS vector (Niwa et al., 1991). The full length and mutant constructs of mouse Tiam 1 and Smo were tagged with HA at their N-termini and subcloned into pCAGGS vector. To generate tagged Smo, HA-tag was inserted within nucleotides 105-106. The truncated constructs TiamF (amino acids 1-400), TiamC (amino acids 393-841), TiamR (amino acids 835-1591) were generated by PCR using the full length cDNA of Tiam1 as a template. SmoCF (amino acids 542-694) and the point mutation in SmoA1 (amino acids W539L), Smo-R (nucleotides G804C, T807A and T810A) and SmoR $\Delta$ CFb (SmoR lacking amino acids 618-713) were generated by PCR using the full length Smo as a 
template. The cDNA fragments of Ptc (nucleotides 1-500) and Smo (130-900) were amplified by

PCR and subcloned into pGEM vectors to generate antisense riboprobes for in situ hybridization.

The mouse Shh cDNA used for in situ hybridization was a gift from E. Laufer.

\section{Cell cultures}

Dissociation cultures of hippocampal neurons were prepared from P0 mouse brains using a neuron dissociation kit (Sumilon). Cells were plated on coverslips coated with poly-d-lysine $(0.4 \mathrm{mg} / \mathrm{ml}$, Sigma) at a density of $10 \times 10^{4}$ cells $/ \mathrm{cm}^{2}$ and maintained in Neurobasal medium supplemented with 1\% B27 supplement (Invitrogen). For transient expression and RNAi experiments, neurons were transfected with plasmids at 7 DIV by using Lipofectamine 2000 (Invitrogen).

NIH3T3 cells were maintained in Dulbecco's Modified Eagle Media (DMEM; Invitrogen) supplemented with $10 \%$ calf serum (GIBCO). For induction of membrane ruffles, cells were starved in a low serum medium for $24 \mathrm{hr}$ and treated with $5 \mu \mathrm{g} / \mathrm{ml}$ Shh for $60 \mathrm{~min}$. In some experiments, 10 $\mu \mathrm{g} / \mathrm{ml}$ actinomycin D (Sigma) was added to the low serum medium $2 \mathrm{hr}$ before Shh addition. HEK293 cells were cultured in DMEM supplemented with 10\% fetal bovine serum (JRH biosciences) and transfected with plasmids using Lipofectamine 2000 (Invitrogen).

\section{Quantification of dendritic protrusions}

Cultured cells were fixed with $4 \%$ paraformaldehyde and permeabilized in $0.2 \%$ Triton X-100 and 5\% BSA in PBS. Samples were processed for immunofluorescence and mounted with ProLong 
Antifade (Molecular Probes). Optical sections of confocal images at $0.5 \mu \mathrm{m}$ z-axis intervals were analyzed using LSM5 Image Browser (Zeiss). The number of dendritic protrusions in thick apical dendrites in the segment of $50 \mu \mathrm{m}$ in length from the point of $30 \mu \mathrm{m}$ away from the cell body was measured. Dendritic spines were defined as protrusions of $0.5-5.0 \mu \mathrm{m}$ in length with large heads and thin necks, whereas filopodia were defined as headless thin protrusions of 0.5-5.0 $\mu \mathrm{m}$ in length. 14-27 neurons from at least three independent cultures were analyzed for each experimental condition.

\section{RNA interference}

To generate vector-based RNAi constructs, BLOCK-iT ${ }^{\mathrm{TM}}$ Pol II miR RNAi Expression Vector Kits (Invitrogen) was used. Double-stranded DNA oligonucleotides including target sequences of Smo (SmoRNAi-1 corresponding to nucleotides 803-823 and SmoRNAi-2 corresponding to nucleotides 1201-1221) and Gli2 (corresponding to nucleotides 3720-3740) were subcloned into pcDNA ${ }^{\mathrm{TM}}$ 6.2-GW/EmGFP-miR vector. The sequence encoded in pcDNA ${ }^{\mathrm{TM}}$ 6.2-GW/EmGFP-miR-neg was utilized for negative control (Invitrogen). For effective expression in neurons, the expression cassettes were transferred into the pCAGGS vector.

\section{In situ hybridization}

In situ hybridization was performed using $12 \mu \mathrm{m}$ fresh-frozen cryosections of P14 mouse brains by a method described previously (Eiraku et al., 2002). Sections were fixed with 4\% paraformaldehyde 
for $15 \mathrm{~min}$ at room temperature, permeated with $1 \mu \mathrm{g} / \mathrm{ml}$ proteinase $\mathrm{K}$ for $1 \mathrm{~min}$, and then probed with a digoxigenin-labeled riboprobe of indicated genes in hybridization solution $(50 \%$ formamide, $2 \times$ SSC, 10 mmTris, $1 \times$ Denhardt's solution, $10 \%$ dextran sulfate, $0.2 \%$ SDS, and $0.5 \mathrm{mg} / \mathrm{ml}$ yeast tRNA) at $60{ }^{\circ} \mathrm{C}$ overnight. After removing excess probes in a series of wash buffers, sections were immunolabeled with alkaline phosphatase-conjugated anti-digoxigenin antibody and then stained with nitro blue tetrazolium/5-bromo-4-chloro-3-indolyl phosphate color substrate.

\section{Immunohistochemistry}

Immunohistochemistry was performed as previously described (Fujishima et al., 2007). Briefly, P14 mice were perfused with $4 \%$ paraformaldehyde. Brains were dissected and immersed in $30 \%$ sucrose in phosphate buffered saline (PBS) overnight at $4^{\circ} \mathrm{C}$. Brains were mounted in OCT Compound (Tissue-Tek, Torrance, CA, USA), cryosectioned at $20 \mu \mathrm{m}$ and subjected to immunostaining with antibodies against Tiam1 (Santa Cruz Biotechnology), MAP2 (Upstate) and DNER (R\&D Systems).

\section{GST-fusion proteins and pull down assays}

The truncated mutants SmoC (amino acids 542-793), SmoCF (amino acids 542-694), SmoCR (amino acids 695-793), SmoCFa (amino acids 542-641), and SmoCFb (amino acids 629-694) were subcloned into pGEX vectors (GE Healthcare). Bacteria expressing these recombinant proteins were lysed with bacteria lysis buffer $(40 \mathrm{mM}$ Tris-HCl, $\mathrm{pH} 7.5,5 \mathrm{mM}$ EDTA, $0.1 \mathrm{mM}$ 
phenylmethylsulfonyl fluoride and 1\% Triton X-100), and GST fusion proteins were purified by glutathione sepharose beads (Amersham). HEK293 cells transfected with indicated constructs were harvested with lysis buffer $(40 \mathrm{mM}$ Tris- $\mathrm{HCl}, \mathrm{pH} 7.5,150 \mathrm{mM} \mathrm{NaCl}, 1 \mathrm{mM}$ EDTA, $1 \mathrm{mM}$ phenylmethylsulfonyl fluoride, $0.5 \%$ Triton X-100 and complete protease inhibitor cocktails). Brains from P14 mice were homogenized in homogenization buffer (50 mM Hepes, pH 7.4, 150 $\mathrm{mM} \mathrm{NaCl}, 2 \mathrm{mM}$ EDTA, $100 \mathrm{mM}$ Sodium Orthovanadate, $1 \mathrm{mM}$ phenylmethylsulfonyl fluoride, 0.1\% SDS, 10\% Glycerol, 1\% NP-40, 0.5\% Deoxycolate and complete protease inhibitor cocktails) in a glass Teflon homogenizer. GST-fusion proteins were immobilized on $50 \mu$ glutathione sepharose beads and then mixed with HEK293 cell lysates or brain extracts. After incubation for 1 hr at $4^{\circ} \mathrm{C}$, the beads were washed, resuspended in SDS sample buffer and subjected for immunoblotting.

\section{Mass spectrometry}

The truncated mutant SmoC (amino acids 542-793) was subcloned into pGEX vectors (GE Healthcare), and GST fusion protein was extracted from bacteria and purified by glutathione sepharose beads (Amersham). Brains from P14 mice were homogenized in homogenization buffer (50 mM Hepes, pH 7.4, 150 mM NaCl, 2 mM EDTA, 100 mM Sodium Orthovanadate, 0.1\% SDS, $10 \%$ Glycerol, $1 \%$ NP-40 and $0.5 \%$ Deoxycolate) in a glass Teflon homogenizer, and mixed with GST-SmoC immobilized on $50 \mu 1$ glutathione sepharose beads. Proteins bound to GST-SmoC were separated by SDS-PAGE and stained with Coomassie blue. Each visible band was excised and 
processed for in-gel trypsin digestion. Collected peptides were then analyzed by the LC-MS/MS system (LTQ, Thermo Electron). The candidate protein sequences were identified by using MASCOT software (Matrix Science).

Mice

Mice used in this study were handled in accordance with the Animal Experiment Committee of the RIKEN Brain Science Institute and Kyoto University and were housed in a specific pathogen-free environment with a $12 \mathrm{hr}$ light/dark cycle. 


\section{Acknowledgments}

We would like to thank Drs. H. Sasaki, A. Miyawaki and E. Laufer for constructs, and Drs. H.

Kamiguchi, J. Taipale and J. Motoyama for invaluable advices. We also thank the Research

Resources Center of RIKEN BSI for technical assistance and all Kengaku lab members for technical help and discussion throughout this work. 


\section{References}

Anton, I.M., Saville, S.P., Byrne, M.J., Curcio, C., Ramesh, N., Hartwig, J.H., Geha, R.S., 2003. WIP participates in actin reorganization and ruffle formation induced by PDGF. Journal of cell science 116, 2443-2451.

Bai, C.B., Auerbach, W., Lee, J.S., Stephen, D., Joyner, A.L., 2002. Gli2, but not Gli1, is required for initial Shh signaling and ectopic activation of the Shh pathway. Development (Cambridge, England) 129, 4753-4761.

Beachy, P.A., Karhadkar, S.S., Berman, D.M., 2004. Tissue repair and stem cell renewal in carcinogenesis. Nature 432, 324-331.

Bijlsma, M.F., Borensztajn, K.S., Roelink, H., Peppelenbosch, M.P., Spek, C.A., 2007. Sonic hedgehog induces transcription-independent cytoskeletal rearrangement and migration regulated by arachidonate metabolites. Cellular signalling 19, 2596-2604.

Buchanan, F.G., Elliot, C.M., Gibbs, M., Exton, J.H., 2000. Translocation of the Rac1 guanine nucleotide exchange factor Tiam1 induced by platelet-derived growth factor and lysophosphatidic acid. The Journal of biological chemistry 275, 9742-9748.

Calabrese, B., Wilson, M.S., Halpain, S., 2006. Development and regulation of dendritic spine synapses. Physiology (Bethesda, Md 21, 38-47.

Chen, J.K., Taipale, J., Cooper, M.K., Beachy, P.A., 2002a. Inhibition of Hedgehog signaling by direct binding of cyclopamine to Smoothened. Genes \& development 16, 2743-2748.

Chen, J.K., Taipale, J., Young, K.E., Maiti, T., Beachy, P.A., 2002b. Small molecule modulation of Smoothened activity. Proceedings of the National Academy of Sciences of the United States of America 99, 14071-14076.

Cingolani, L.A., Goda, Y., 2008. Actin in action: the interplay between the actin cytoskeleton and synaptic efficacy. Nature reviews 9, 344-356.

Eiraku, M., Hirata, Y., Takeshima, H., Hirano, T., Kengaku, M., 2002. Delta/notch-like epidermal growth factor (EGF)-related receptor, a novel EGF-like repeat-containing protein targeted to dendrites of developing and adult central nervous system neurons. The Journal of biological chemistry $277,25400-25407$.

Ethell, I.M., Pasquale, E.B., 2005. Molecular mechanisms of dendritic spine development and remodeling. Progress in neurobiology 75, 161-205.

Feinmark, S.J., Begum, R., Tsvetkov, E., Goussakov, I., Funk, C.D., Siegelbaum, S.A., Bolshakov, V.Y., 2003. 12-lipoxygenase metabolites of arachidonic acid mediate metabotropic glutamate receptor-dependent long-term depression at hippocampal CA3-CA1 synapses. J Neurosci 23, 11427-11435.

Fuccillo, M., Joyner, A.L., Fishell, G., 2006. Morphogen to mitogen: the multiple roles of hedgehog signalling in vertebrate neural development. Nature reviews 7, 772-783.

Fujishima, K., Kiyonari, H., Kurisu, J., Hirano, T., Kengaku, M., 2007. Targeted disruption of Sept3, 
a heteromeric assembly partner of Sept5 and Sept7 in axons, has no effect on developing CNS neurons. Journal of neurochemistry 102, 77-92.

Hammond, R., Blaess, S., Abeliovich, A., 2009. Sonic hedgehog is a chemoattractant for midbrain dopaminergic axons. PloS one 4, e7007.

Huangfu, D., Anderson, K.V., 2006. Signaling from Smo to Ci/Gli: conservation and divergence of Hedgehog pathways from Drosophila to vertebrates. Development (Cambridge, England) 133, 3-14. Jaffe, A.B., Hall, A., 2005. Rho GTPases: biochemistry and biology. Annual review of cell and developmental biology 21, 247-269.

Kawauchi, T., Chihama, K., Nabeshima, Y., Hoshino, M., 2003. The in vivo roles of STEF/Tiam1, Rac1 and JNK in cortical neuronal migration. The EMBO journal 22, 4190-4201.

Kovacs, J.J., Whalen, E.J., Liu, R., Xiao, K., Kim, J., Chen, M., Wang, J., Chen, W., Lefkowitz, R.J., 2008. Beta-arrestin-mediated localization of smoothened to the primary cilium. Science (New York, N.Y 320, 1777-1781.

Matsuno, H., Okabe, S., Mishina, M., Yanagida, T., Mori, K., Yoshihara, Y., 2006. Telencephalin slows spine maturation. J Neurosci 26, 1776-1786.

McMahon, A.P., Ingham, P.W., Tabin, C.J., 2003. Developmental roles and clinical significance of hedgehog signaling. Current topics in developmental biology 53, 1-114.

Meloni, A.R., Fralish, G.B., Kelly, P., Salahpour, A., Chen, J.K., Wechsler-Reya, R.J., Lefkowitz, R.J., Caron, M.G., 2006. Smoothened signal transduction is promoted by G protein-coupled receptor kinase 2. Molecular and cellular biology 26, 7550-7560.

Michiels, F., Habets, G.G., Stam, J.C., van der Kammen, R.A., Collard, J.G., 1995. A role for Rac in Tiam1-induced membrane ruffling and invasion. Nature 375, 338-340.

Niwa, H., Yamamura, K., Miyazaki, J., 1991. Efficient selection for high-expression transfectants with a novel eukaryotic vector. Gene 108, 193-199.

Rohatgi, R., Milenkovic, L., Corcoran, R.B., Scott, M.P., 2009. Hedgehog signal transduction by Smoothened: pharmacologic evidence for a 2-step activation process. Proceedings of the National Academy of Sciences of the United States of America 106, 3196-3201.

Rohatgi, R., Milenkovic, L., Scott, M.P., 2007. Patched1 regulates hedgehog signaling at the primary cilium. Science (New York, N.Y 317, 372-376.

Rotrosen, D., Gallin, J.I., 1986. Histamine type I receptor occupancy increases endothelial cytosolic calcium, reduces F-actin, and promotes albumin diffusion across cultured endothelial monolayers. The Journal of cell biology 103, 2379-2387.

Ruiz i Altaba, A., Palma, V., Dahmane, N., 2002. Hedgehog-Gli signalling and the growth of the brain. Nature reviews 3, 24-33.

Salter, M.W., Kalia, L.V., 2004. Src kinases: a hub for NMDA receptor regulation. Nature reviews 5, 317-328.

Saneyoshi, T., Wayman, G., Fortin, D., Davare, M., Hoshi, N., Nozaki, N., Natsume, T., Soderling, T.R., 2008. Activity-dependent synaptogenesis: regulation by a CaM-kinase kinase/CaM-kinase I/betaPIX signaling complex. Neuron 57, 94-107.

Schubert, V., Dotti, C.G., 2007. Transmitting on actin: synaptic control of dendritic architecture. 
Journal of cell science 120, 205-212.

Stam, J.C., Sander, E.E., Michiels, F., van Leeuwen, F.N., Kain, H.E., van der Kammen, R.A., Collard, J.G., 1997. Targeting of Tiam1 to the plasma membrane requires the cooperative function of the N-terminal pleckstrin homology domain and an adjacent protein interaction domain. The Journal of biological chemistry 272, 28447-28454.

Taipale, J., Chen, J.K., Cooper, M.K., Wang, B., Mann, R.K., Milenkovic, L., Scott, M.P., Beachy, P.A., 2000. Effects of oncogenic mutations in Smoothened and Patched can be reversed by cyclopamine. Nature 406, 1005-1009.

Tolias, K.F., Bikoff, J.B., Burette, A., Paradis, S., Harrar, D., Tavazoie, S., Weinberg, R.J., Greenberg, M.E., 2005. The Rac1-GEF Tiam1 couples the NMDA receptor to the activity-dependent development of dendritic arbors and spines. Neuron 45, 525-538.

Tolias, K.F., Bikoff, J.B., Kane, C.G., Tolias, C.S., Hu, L., Greenberg, M.E., 2007. The Rac1 guanine nucleotide exchange factor Tiam1 mediates EphB receptor-dependent dendritic spine development. Proceedings of the National Academy of Sciences of the United States of America 104, 7265-7270.

Varjosalo, M., Taipale, J., 2008. Hedgehog: functions and mechanisms. Genes \& development 22, 2454-2472.

Wilson, C.W., Chen, M.H., Chuang, P.T., 2009. Smoothened adopts multiple active and inactive conformations capable of trafficking to the primary cilium. PloS one 4, e5182.

Yam, P.T., Langlois, S.D., Morin, S., Charron, F., 2009. Sonic hedgehog guides axons through a noncanonical, Src-family-kinase-dependent signaling pathway. Neuron 62, 349-362.

Zavros, Y., Orr, M.A., Xiao, C., Malinowska, D.H., 2008. Sonic hedgehog is associated with $\mathrm{H}+-\mathrm{K}+-\mathrm{ATPase}-\mathrm{containing}$ membranes in gastric parietal cells and secreted with histamine stimulation. American journal of physiology 295, G99-G111.

Zhang, H., Macara, I.G., 2006. The polarity protein PAR-3 and TIAM1 cooperate in dendritic spine morphogenesis. Nature cell biology 8, 227-237.

Zhao, Y., Tong, C., Jiang, J., 2007. Hedgehog regulates smoothened activity by inducing a conformational switch. Nature 450, 252-258. 


\section{Figure legends}

\section{Fig. 1. Expression of Shh pathway molecules in the cerebellum and hippocampus.}

(A) Cryosections along the sagittal plane of a postnatal day 14 (P14) mouse brain processed for in situ hybridization with a Smo antisense riboprobe (left) and sense control riboprobe (right). (B) In situ hybridization was carried out on sagittal sections of P14 mouse brain with antisense riboprobes for Shh (left), Ptc (middle) and Smo (right). Expression in the cerebellum and hippocampus were shown in upper and lower panels, respectively. ML, Molecular layer; PL, Purkinje cell layer; IGL, Internal granule layer. Scale bars: $2 \mathrm{~mm}$ in (A); $10 \mu \mathrm{m}$ in upper panels in (B); $100 \mu \mathrm{m}$ in lower panels in (B).

\section{Fig. 2. The Shh pathway is implicated in spine formation}

(A) Hippocampal neurons were transfected with Venus to visualize cell shape. Spines and filopodia were measured at 16 DIV after treatment with PBS (a) or $5 \mu \mathrm{g} / \mathrm{ml}$ Shh (b) from 8 to $16 \mathrm{DIV}$, or with DMSO (c), $5 \mu \mathrm{M}$ cyclopamine (d) or $100 \mathrm{nM}$ SANT-1 (e) from 15 to 16 DIV. Arrow heads and arrows in (a) indicate spines and filopodia, respectively. (B) The numbers of spines and filopodia per unit length on apical dendrites of hippocampal neurons described in A were quantified. (C) Hippocampal neurons were transfected with indicated constructs (a-d) at 7 DIV and observed at 16 DIV. (D) The numbers of spines and filopodia per unit length on apical dendrites of hippocampal 
neurons described in $\mathrm{C}$ were quantified. Values represent mean \pm S.E.M. ${ }^{*} \mathrm{P}<0.001, * * \mathrm{P}<0.005$, Student's t test in (B), (D). Scale bars represent $5 \mu \mathrm{m}$ in (A), (C).

\section{Fig. 3. Smo interacts with the Rac1 GEF Tiam1}

(A) (Left) Pull down assays from P14 mouse brain extracts were performed using GST or GST-SmoC. Bound proteins were separated by SDS-PAGE using 10\% gel. Several signals could be seen especially in the higher molecular weight. (Right) GST or GST-SmoC binding proteins were separated by SDS-PAGE using 7.5\% gel and analyzed by mass spectrometry. Identified molecules are shown on the right. Numbers on the left indicate molecular weight. (B) GST-SmoC or GST was incubated with P14 mouse brain extracts, and bound proteins were identified by immunoblot analyses with the indicated antibodies. Two percent of lysate used for pull down assays was applied to the control lane. The expression of GST-SmoC and GST proteins were verified by Coomassie blue staining in lower panel. (C) Immunofluorescence of P14 mouse hippocampus with antibodies against Tiam1 (a, d) and DNER (b, e). Merged views are shown in c, f. Tiam1 was expressed in neurons in CA1 to CA3 and in the dentate gyrus. Higher magnified view reveals punctate expression of Tiam1 in the somata and along apical dendrites (g, $\mathrm{i}$; green) demarcated by MAP2 expression (h, i; red). Scale bars: $10 \mu \mathrm{m}$ in a-c; $50 \mu \mathrm{m}$ in d-f; $200 \mu \mathrm{m}$ in g-i.

\section{Fig. 4. Binding domains of Smo and Tiam1}


(A) Schematic representation of Tiam1, Smo C-terminal tail, and respective deletion constructs. $\mathrm{PH}$, pleckstrin homology domain; RBD, Ras-binding domain; PDZ, PSD95/DLG/zo-1 domain; Rho GEF, guanine nucleotide exchange factor for Rho GTPases; PB, poly-basic region; TM, trans-membrane region. The results of GST pull down assays are summarized on the right. (B)

GST-SmoC was incubated with cell lysate expressing HA-tagged full length or deletion mutants of Tiam1. Interactions were examined by immunoblot analysis with an anti-HA antibody. GST-SmoC proteins were visualized by Coomassie blue staining in lower panel. (C) Left; GST, GST-SmoC, GST-SmoCF and GST-SmoCR were incubated with cell lysate expressing HA-tagged full length Tiam1. Right; GST pull down assays with the anterior and posterior fragments of SmoCF (GST-SmoCFa and GST-SmoCFb). Interactions were examined by immunoblot analysis with an anti-HA antibody. GST and GST-fused proteins were visualized by Coomassie blue staining in lower panels.

\section{Fig. 5. Shh regulates actin cytoskeleton remodeling}

(A) Shh induces membrane ruffles in NIH3T3 cells. NIH3T3 cells were serum-starved for $24 \mathrm{hr}$ and then treated with or without $5 \mu \mathrm{g} / \mathrm{ml} \mathrm{Shh}$ for $60 \mathrm{~min}(\mathrm{a}, \mathrm{b})$. Cells in the right panels were pretreated with $10 \mu \mathrm{g} / \mathrm{ml}$ actinomycin D for $2 \mathrm{hr}$ before addition of Shh (c, d). Actin filaments were stained with rhodamine-phalloidin. Lower panels are higher magnified views of boxed regions in respective upper panels (e-h). (B) The numbers of cells bearing membrane ruffling were quantified. The number in parenthesis indicates the total number of cells scored. $* \mathrm{P}<0.001$, chi-square test. NS 
indicates not significant $(\mathrm{P}>0.5)$. (C) Tiam1 DN suppressed membrane ruffles induced by Shh. Cells were transfected with Tiam1 DN, serum-starved, and then treated with $5 \mu \mathrm{g} / \mathrm{ml} \mathrm{Shh}$ for $60 \mathrm{~min}$. Cells were fixed and stained with anti-HA (left) and rhodamine-phalloidin (right) to detect Tiam1 DN and actin filaments, respectively. Shh stimulated membrane ruffles in cells that did not express Tiam1 DN (arrowheads), but not in the cell expressing high levels of Tiam1 DN (arrows). (D) Shh induces translocation of Tiam1 to the plasma membrane. Serum-starved cells were treated with or without $5 \mu \mathrm{g} / \mathrm{ml}$ Shh for $60 \mathrm{~min}$ and then fixed and stained with rhodamine-phalloidin $(\mathrm{a}, \mathrm{b})$ and an anti-Tiam1 antibody (c, d). Boxed regions in upper panels are enlarged in lower panels (e-j). Scale bars in (A), (C) and (D) represent $10 \mu \mathrm{m}$.

\section{Fig. 6. Shh induces spine formation through the Tiam1-Rac1 pathway}

(A) Schematic representation of mutant constructs for Tiam1 and Smo. (B) Hippocampal neurons transfected with Venus were treated with $50 \mu \mathrm{M}$ Rac1 inhibitor for $12 \mathrm{hr}$ (c) or were co-transfected with Tiam1 DN (b), SmoCF (d) or SmoA1 (e). In the right columns, neurons transfected with SmoRNAi-1 (f) were co-transfected with Tiam1 DN (g) or SmoR $\Delta$ CFb (i), or treated with Rac1 inhibitor (h). (C) The numbers of spines and filopodia per unit length on apical dendrites of hippocampal neurons described in A were quantified. Values represent mean \pm S.E.M. $* \mathrm{P}<0.001$, Student's t test. Scale bar in (A) represent $5 \mu \mathrm{m}$.

Fig. 7. Model of Shh signaling pathway regulating the actin cytoskeleton 
Inactive juvenile Smo (0) matures and binds to Tiam1 in the absence of Shh (I). Shh binds to Ptc and relieves the inhibitory effect of Ptc on Smo (II). Smo becomes active and transduces the Shh signal to intracellular pathways including Gli-mediated transcription. Activated Smo loses its Tiam1-binding activity, and Tiam1 is thus released and induces reorganization of the actin cytoskeleton via Rac1. Putative inhibitory functions of SANT-1 and cyclopamine are indicated. 
A

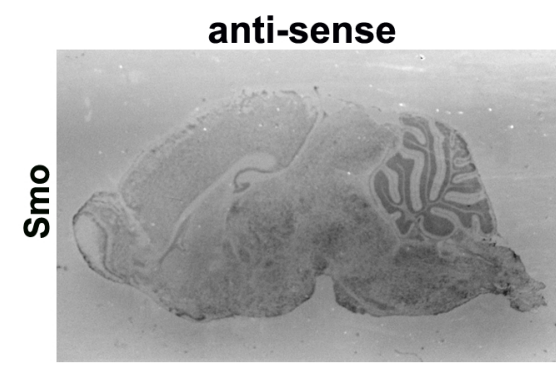

sense

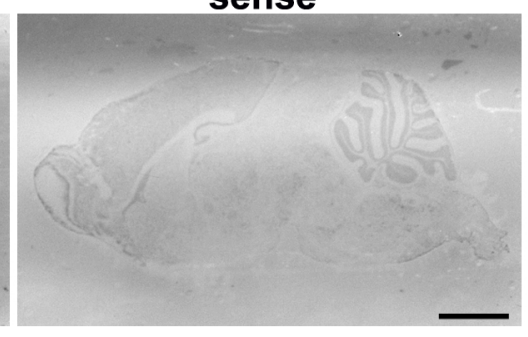

B
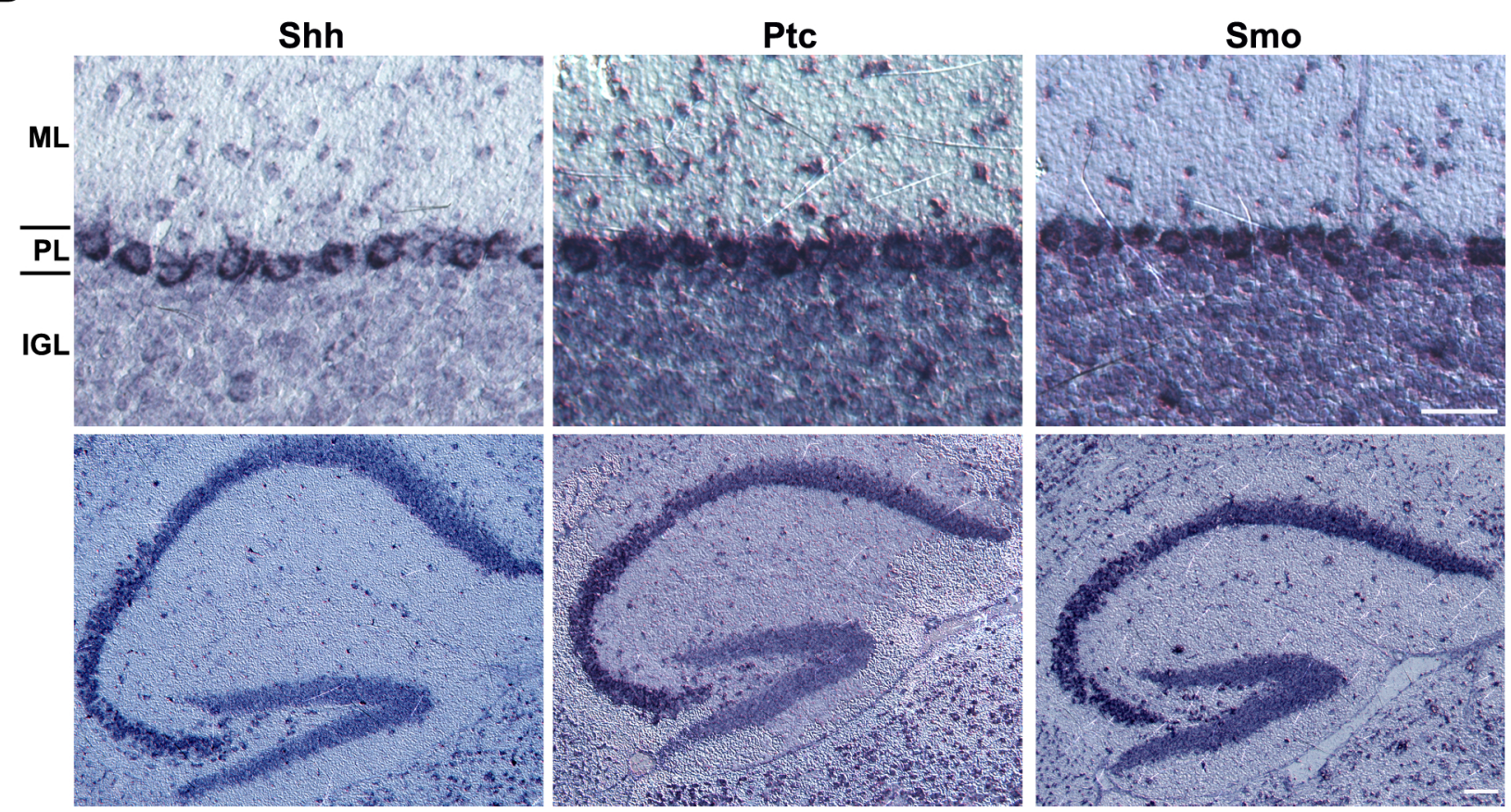
A
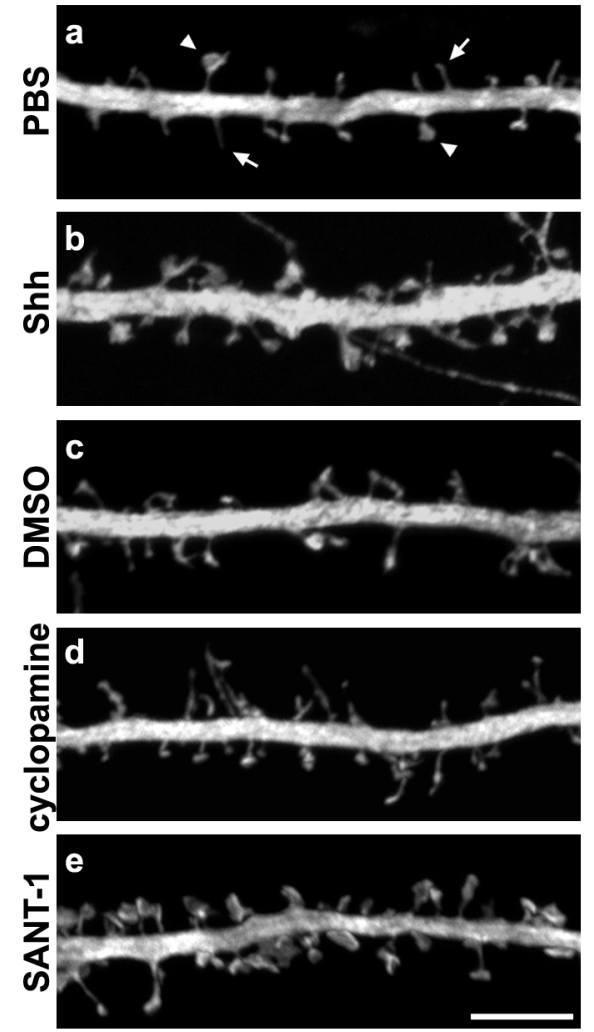

C
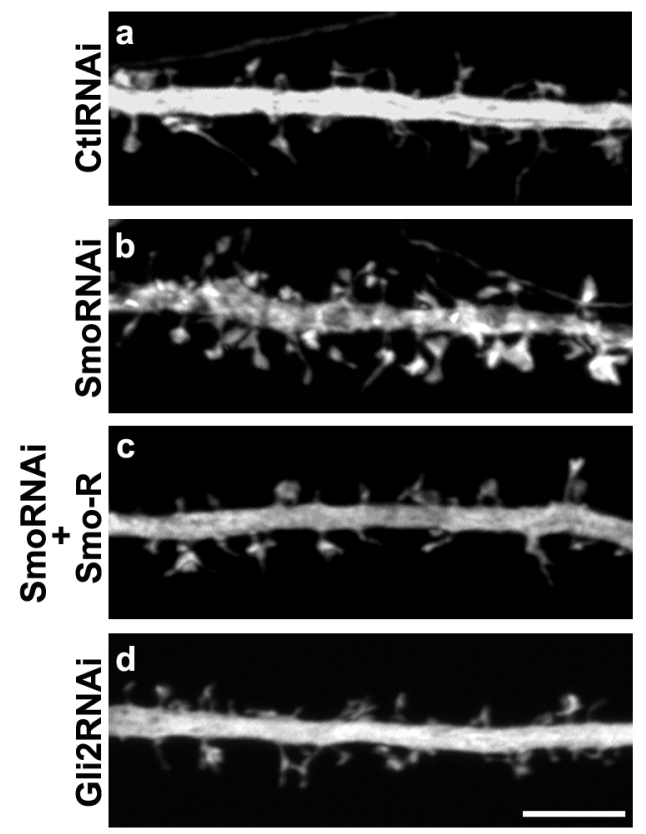

B

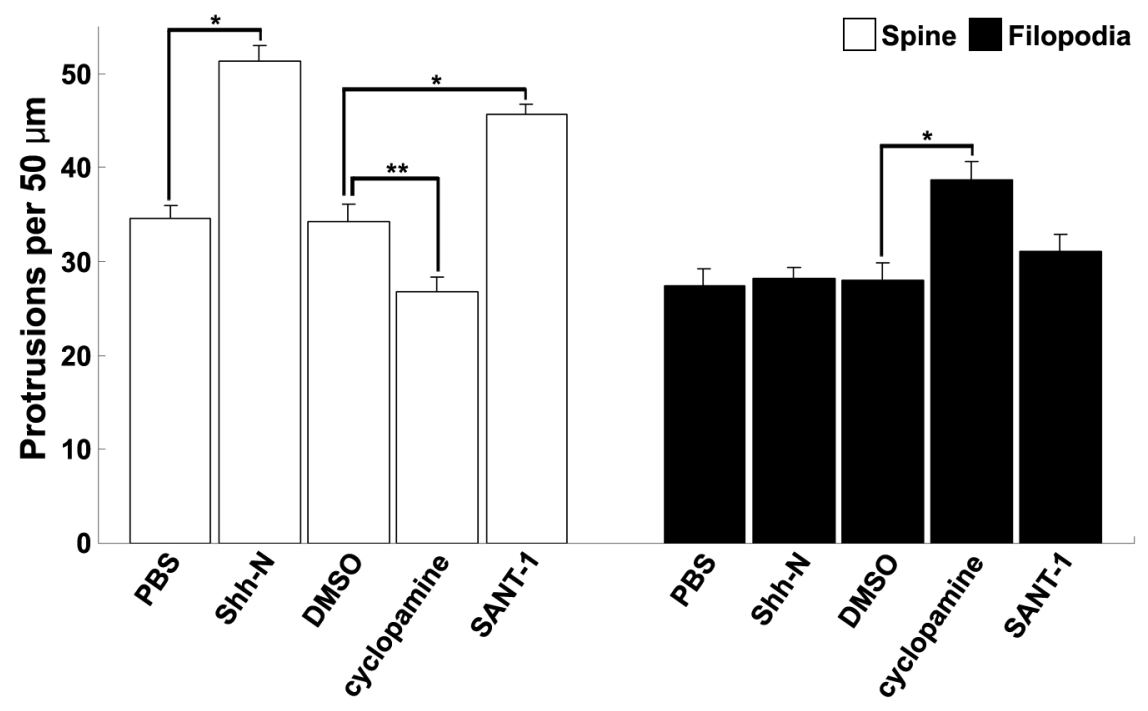

D

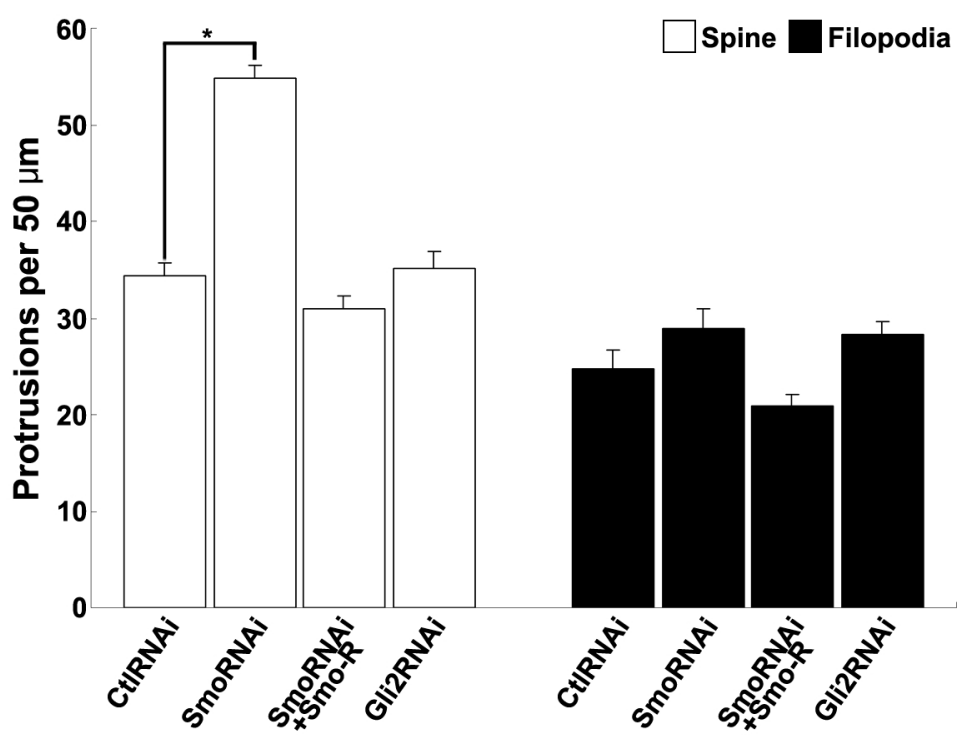

Figure 2 
A

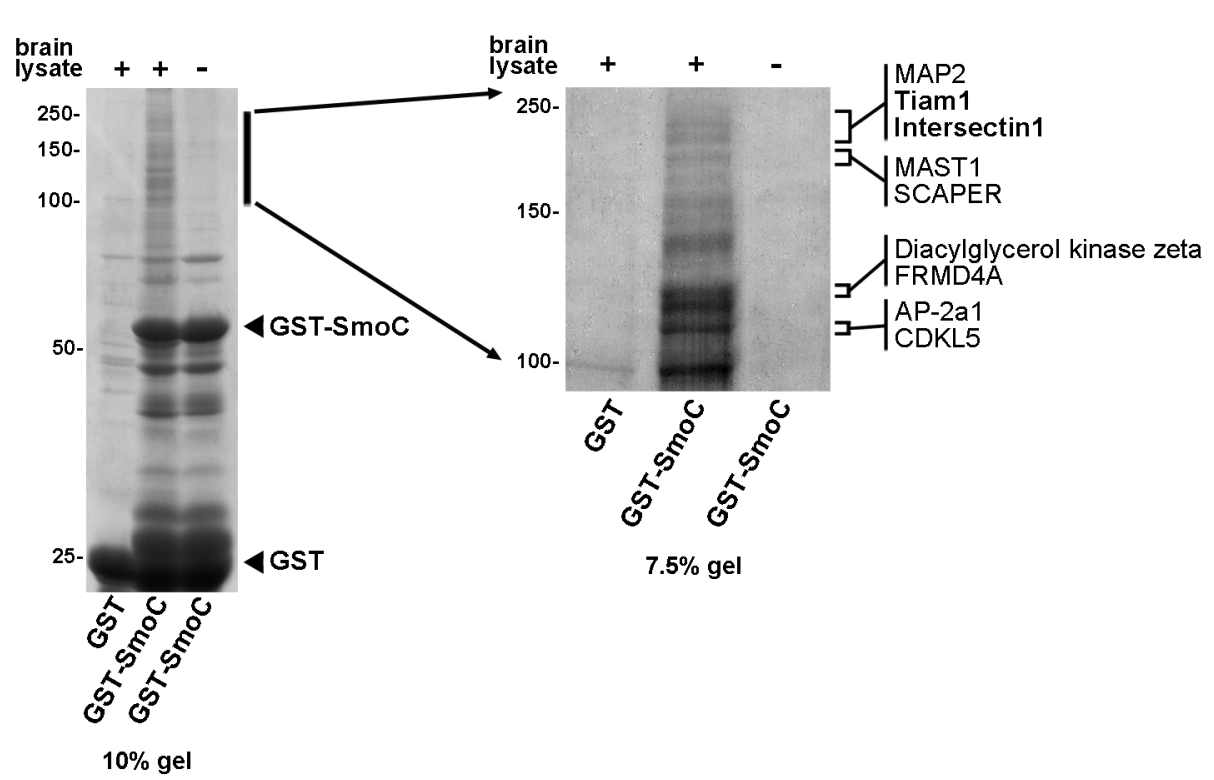

B

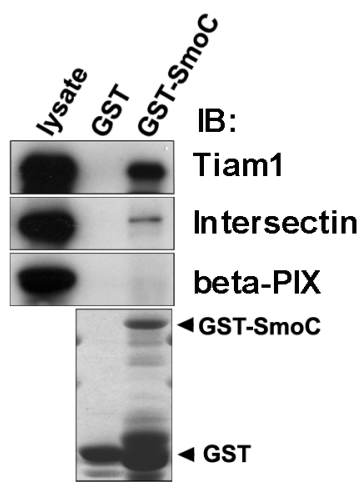

C

Tiam1
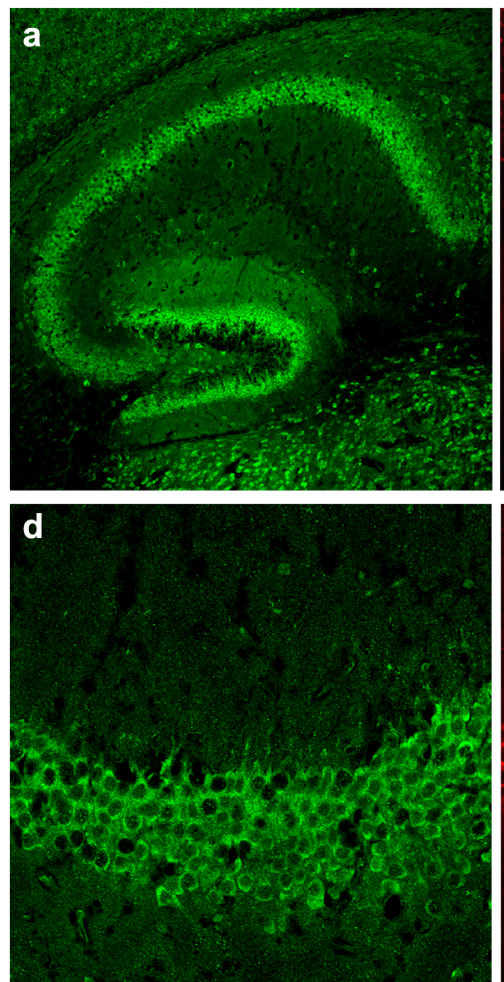

Tiam1

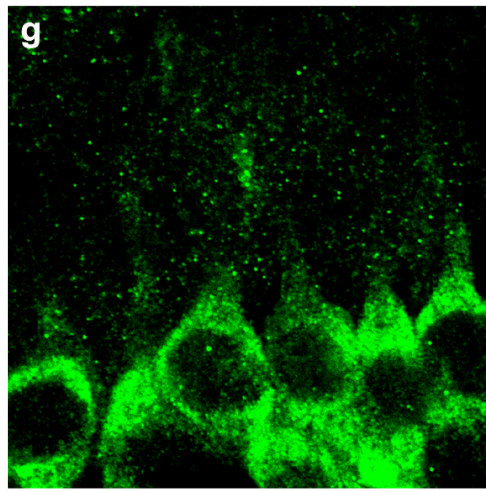

DNER
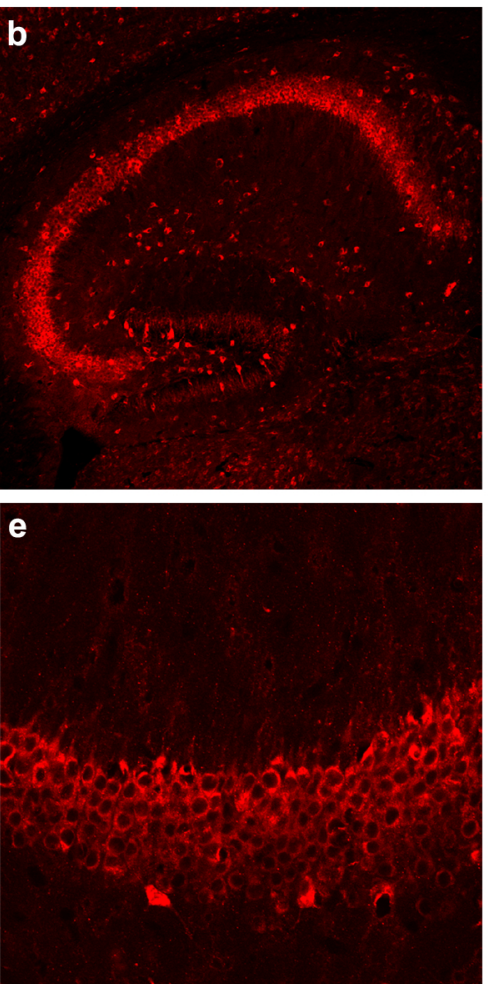

MAP2

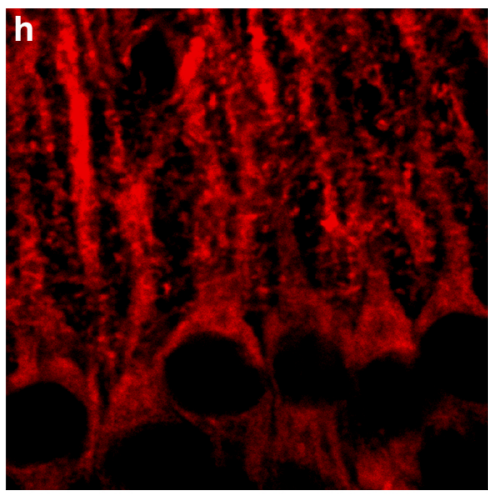

Merge
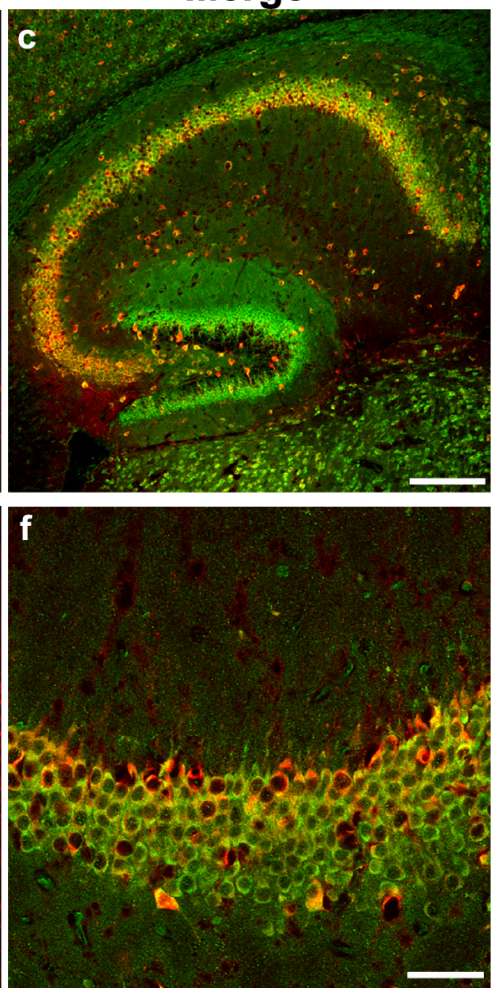

Merge

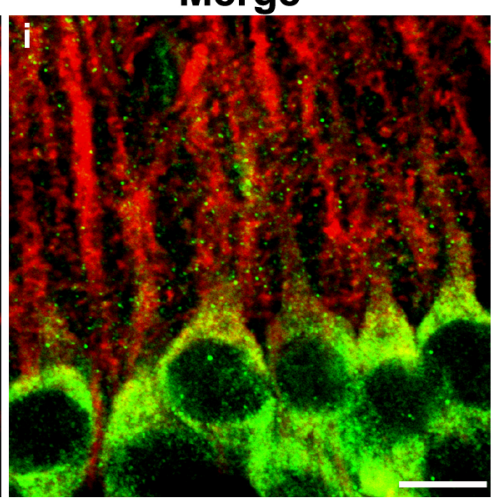


A
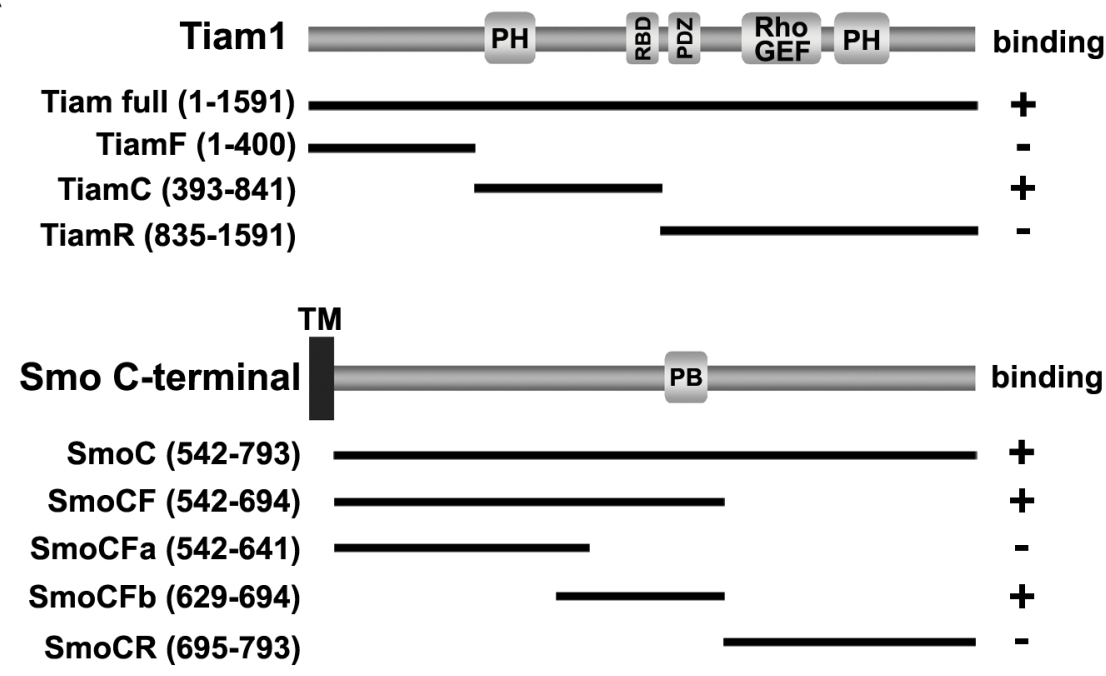

B

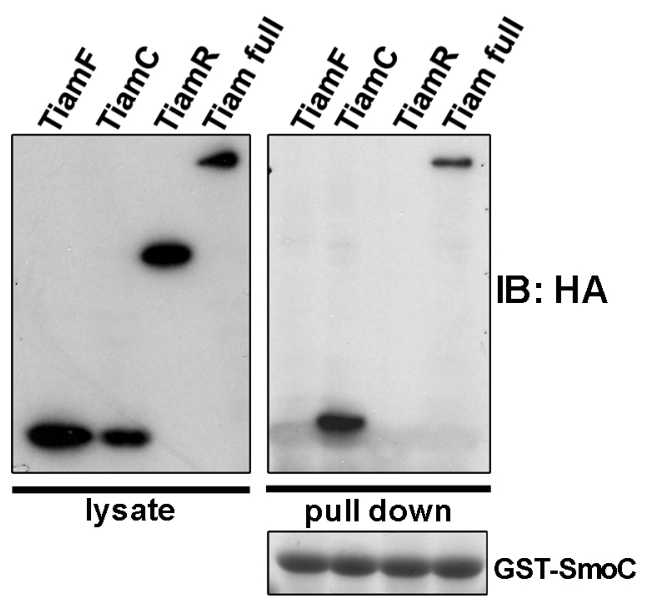

C

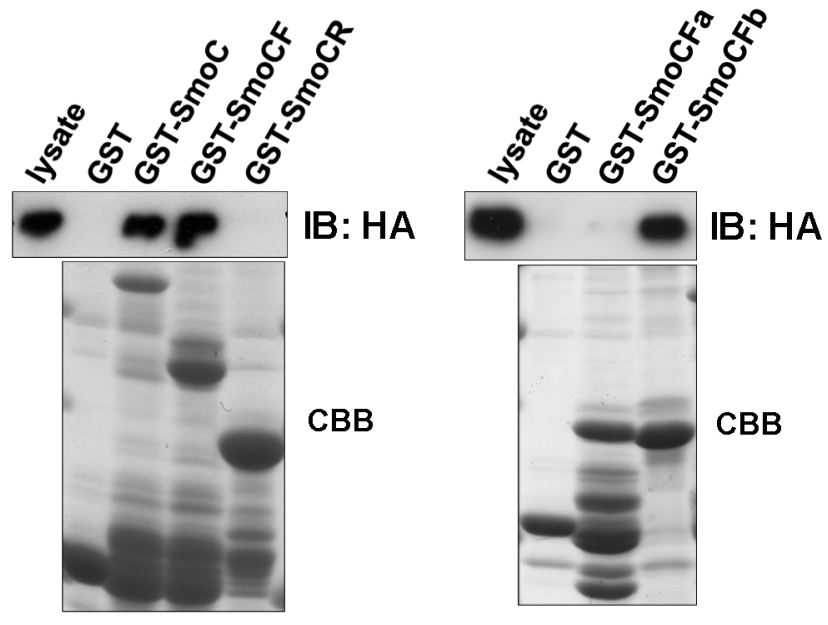


A
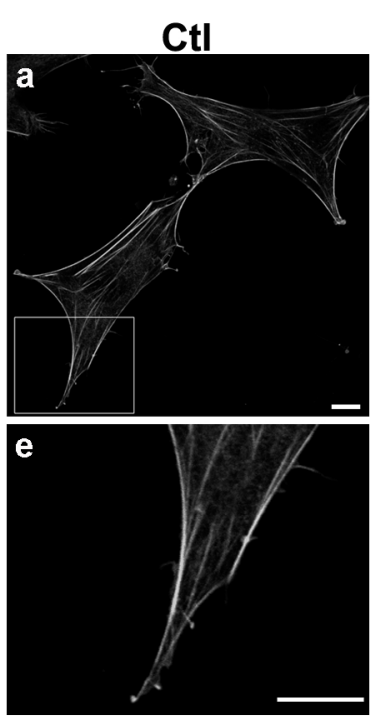

B

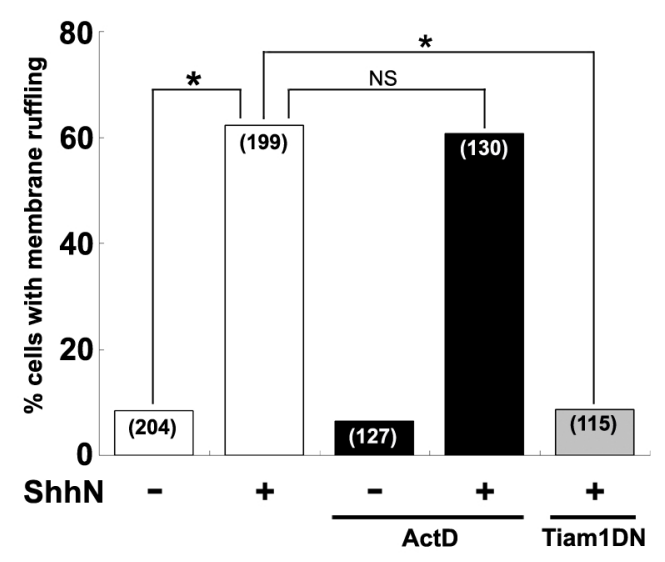

C

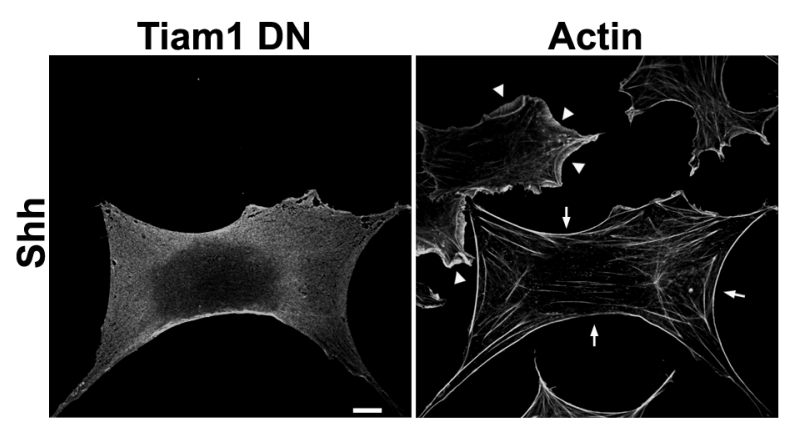

Actinomycin D

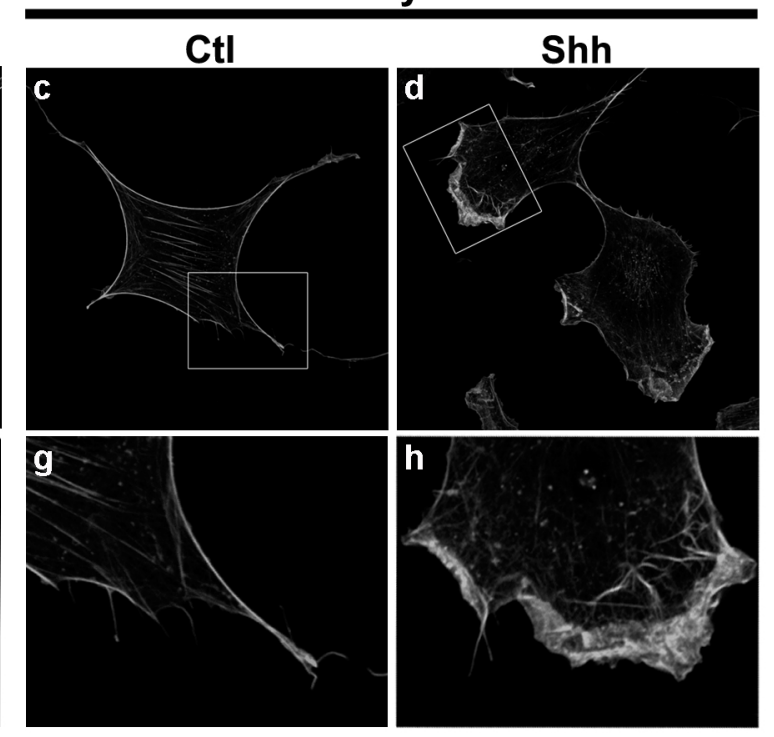

D
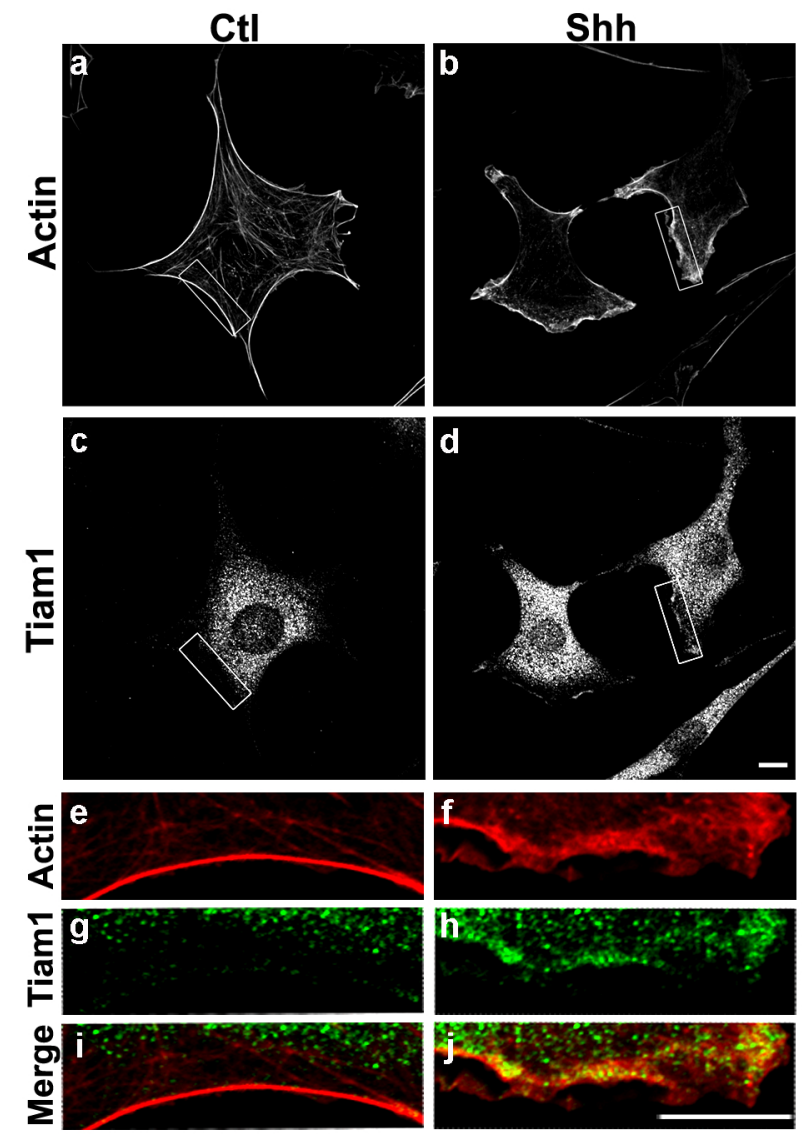


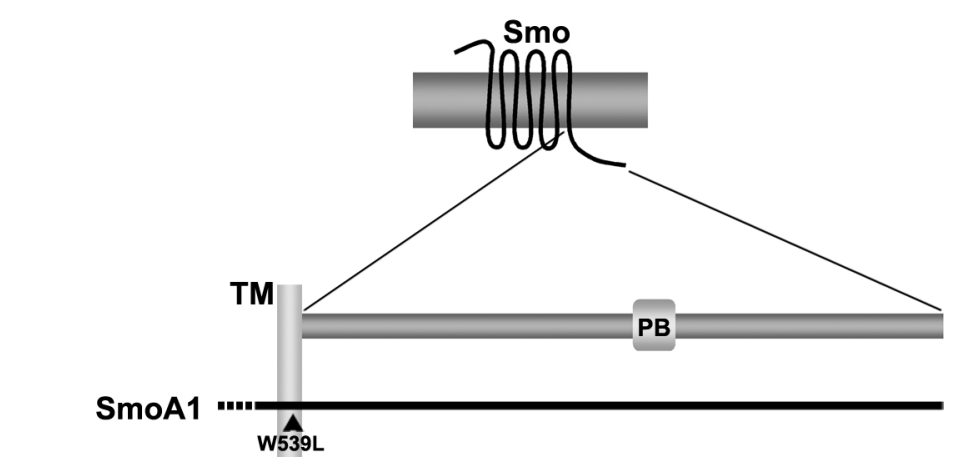

Tiam1 $\mathrm{PH}$ 受-

Tiam1DN (TiamC) 841

SmoR $\triangle$ CFb

SmoCF

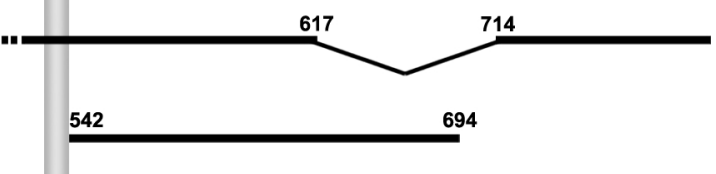

B
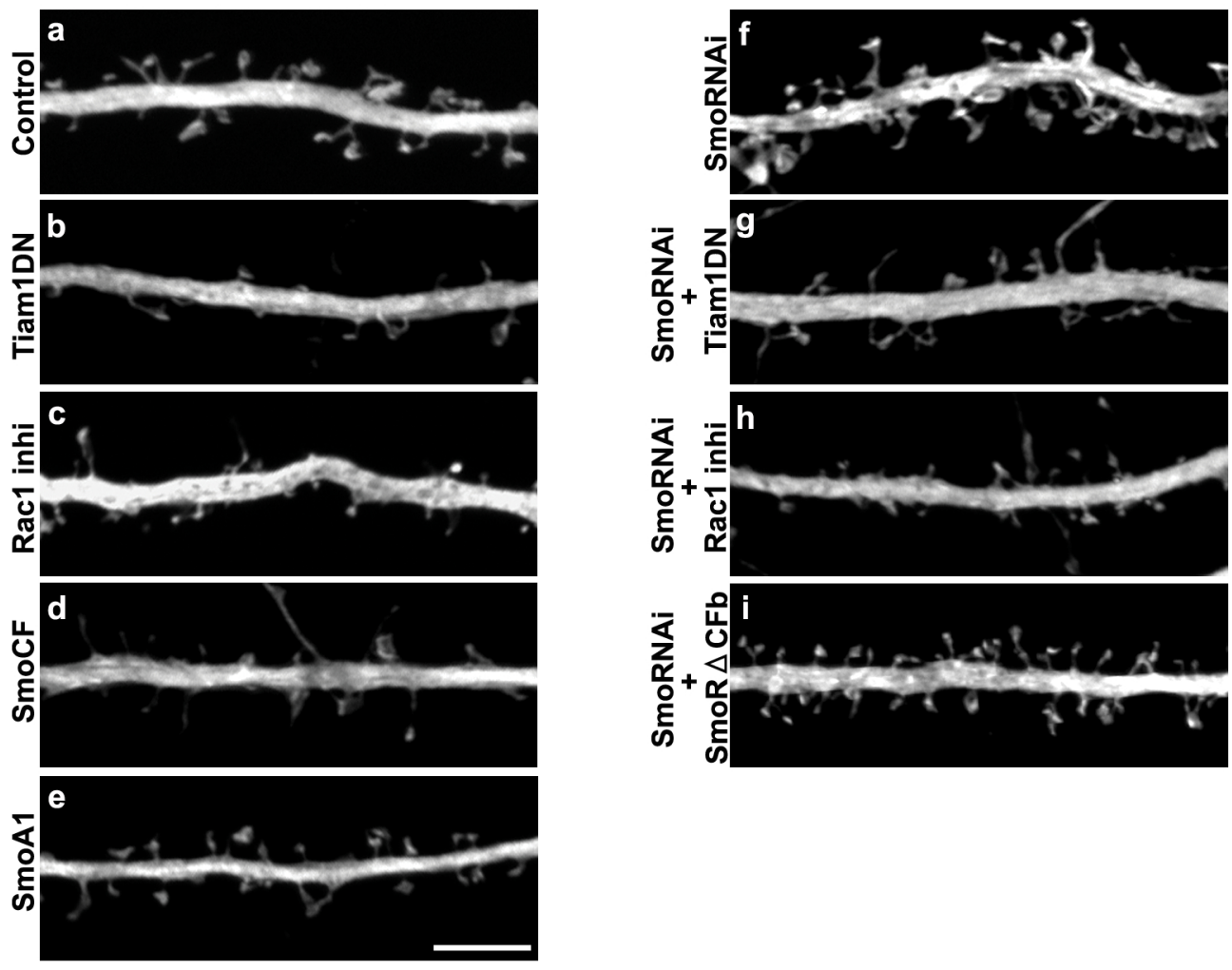

C

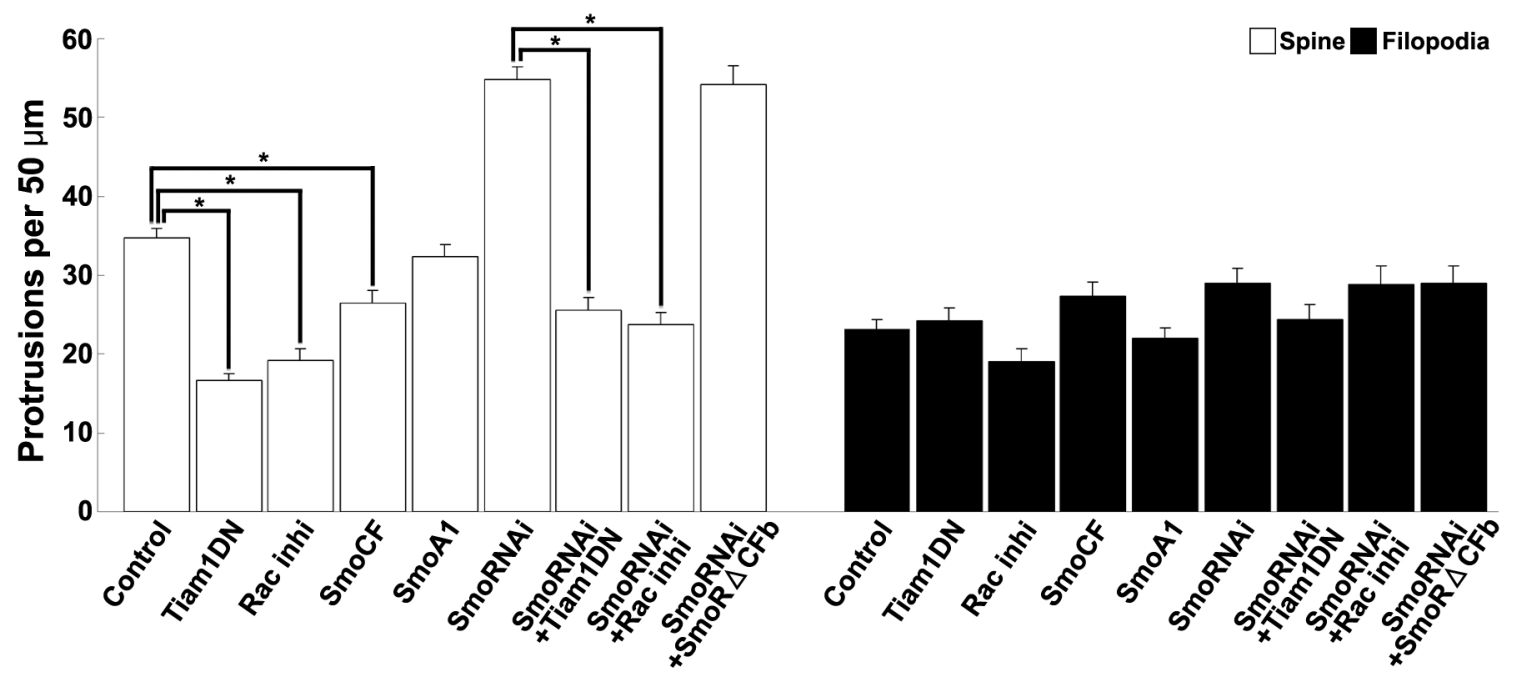

Figure 6 


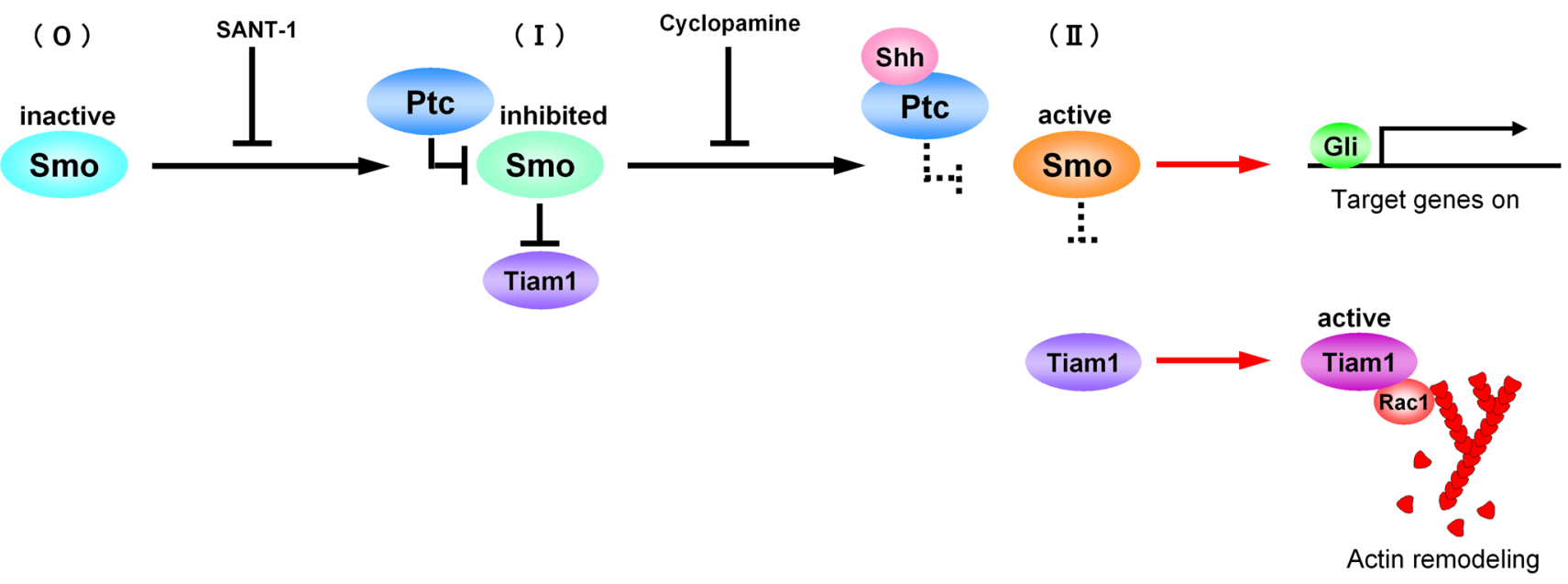




\title{
Supplementary material
}

\section{Sonic hedgehog signaling regulates actin cytoskeleton via Tiam1-Rac1 cascade during spine formation}

\author{
Nobunari Sasaki, Junko Kurisu and Mineko Kengaku
}

\section{Supplemental Experimental Methods}

\section{Reporter assay}

NIH3T3 cells were transfected with $8 \times 3$ ' Gli-BS-luciferase (a gift from H. Sasaki) (Sasaki et al., 1997; Sasaki et al., 1999), Renilla luciferase (pRL-TK, Promega) and the indicated constructs using Lipofectamine LTX (Invitrogen). At $48 \mathrm{hr}$ after transfection, culture medium was replaced with fresh medium containing low serum $(0.5 \%$ calf serum $)$ and incubated for an additional $24 \mathrm{hr}$. Cells were then treated with Shh with or without $5 \mu \mathrm{M}$ cyclopamine or $100 \mathrm{nM}$ SANT-1 and cultured for 3 days. Luciferase assays were performed using the PiccaGene Dual Sea Pansy Luciferase Assay kit (Toyo Ink) following the manufacturer's protocol. Values were normalized using Renilla luciferase activity.

\section{Dissociation culture of cerebellar neurons}

Dissociation cultures of cerebellar neurons were prepared from P0 mouse brains using a neuron dissociation kit (Sumilon). Neurons were plated on coverslips coated with poly-d-lysine and maintained in D-MEM/F-12 medium (Invitrogen) supplemented with $100 \mu \mathrm{M}$ putrescine, $30 \mathrm{nM}$ 
$\mathrm{Na}_{2} \mathrm{SeO}_{3}, 1.4 \mathrm{mM}$ L-glutamine, $200 \mu \mathrm{g} / \mathrm{ml}$ transferrin, $40 \mathrm{nM}$ progesteron and $20 \mu \mathrm{g} / \mathrm{ml}$ Insulin

(Tabata et al., 2000). 


\section{References}

Sasaki, H., Hui, C., Nakafuku, M., Kondoh, H., 1997. A binding site for Gli proteins is essential for HNF-3beta floor plate enhancer activity in transgenics and can respond to Shh in vitro. Development (Cambridge, England) 124, 1313-1322.

Sasaki, H., Nishizaki, Y., Hui, C., Nakafuku, M., Kondoh, H., 1999. Regulation of Gli2 and Gli3 activities by an amino-terminal repression domain: implication of Gli2 and Gli3 as primary mediators of Shh signaling. Development (Cambridge, England) 126, 3915-3924.

Tabata, T., Sawada, S., Araki, K., Bono, Y., Furuya, S., Kano, M., 2000. A reliable method for culture of dissociated mouse cerebellar cells enriched for Purkinje neurons. Journal of neuroscience methods $104,45-53$. 


\section{SUPPLEMENTAL FIGURES}

A
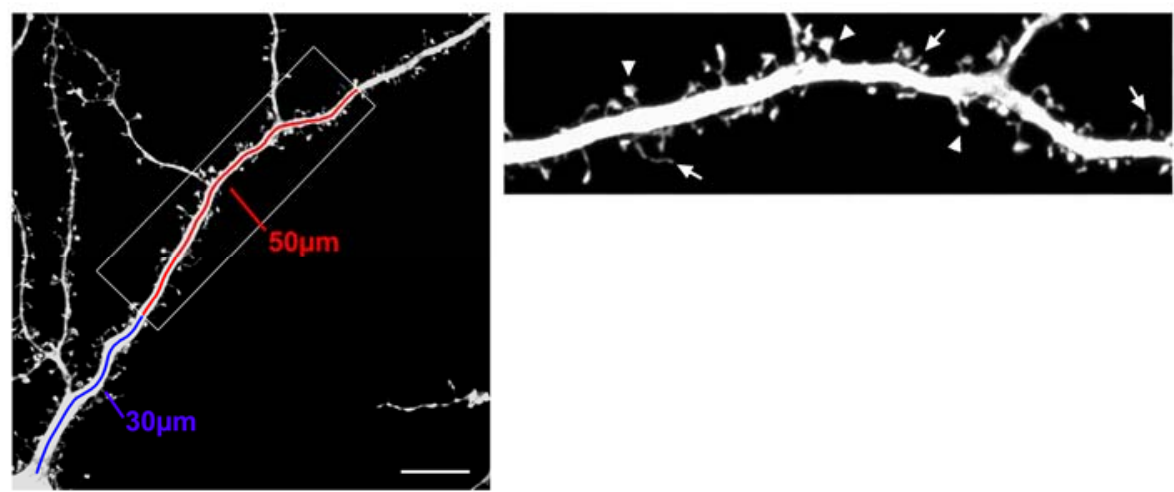

B

C
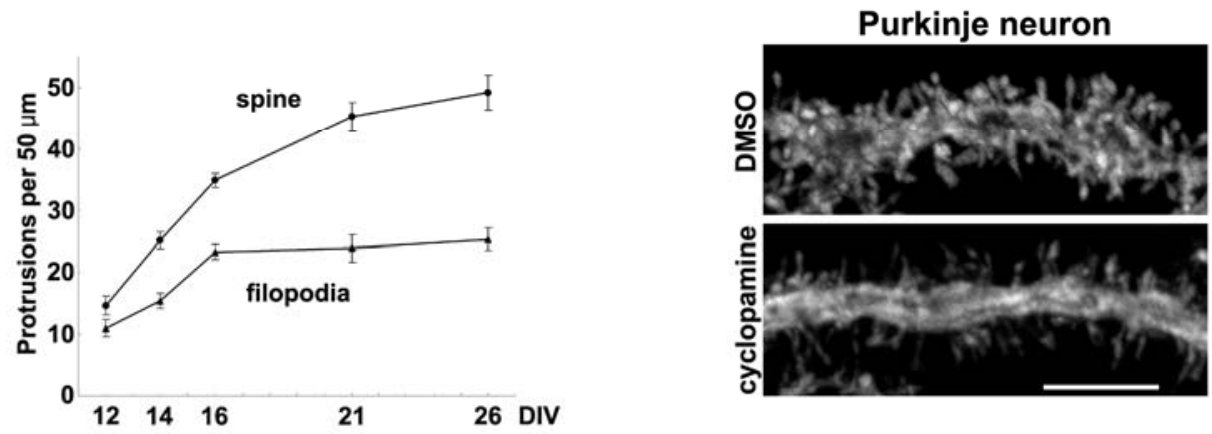

Fig. S1. Analysis of dendritic protrusions in cultured hippocampal neurons

(A) (Left) confocal microscopic image of a hippocampal neuron in primary culture. The number of spines and filopodia in thick apical dendrites in the segment $50 \mu \mathrm{m}$ in length beginning $30 \mu \mathrm{m}$ from the cell body were measured (boxed area). (Right) spine (arrow heads) and filopodia (arrows) are designated in the enlarged view of the boxed area in the left panel. Scale bar represents $10 \mu \mathrm{m}$. (B) The density of dendritic protrusions in cultured hippocampal neurons at different developmental 
stages. (C) Cyclopamine also retards spine formation in cerebellar Purkinje neurons. Purkinje neurons in dissociation culture were treated with either DMSO or cyclopamine from 13 DIV to 14 DIV. Cells were then fixed and stained with anti-calbindin antibody. Cyclopamine treatment increased the density of thin, headless filopodia at the expense of mushroom-shaped spines. Scale bar represents $5 \mu \mathrm{m}$. 
A

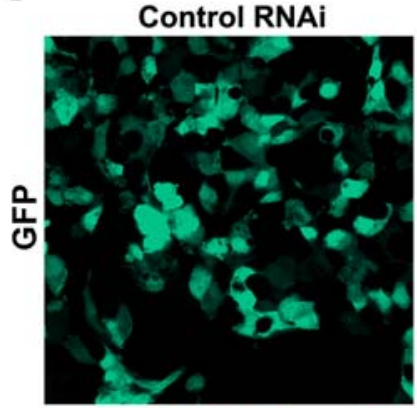

SmoRNAi-1

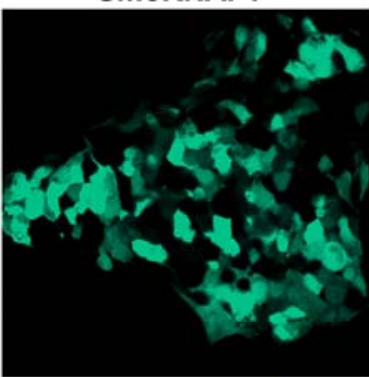

SmoRNAi-2
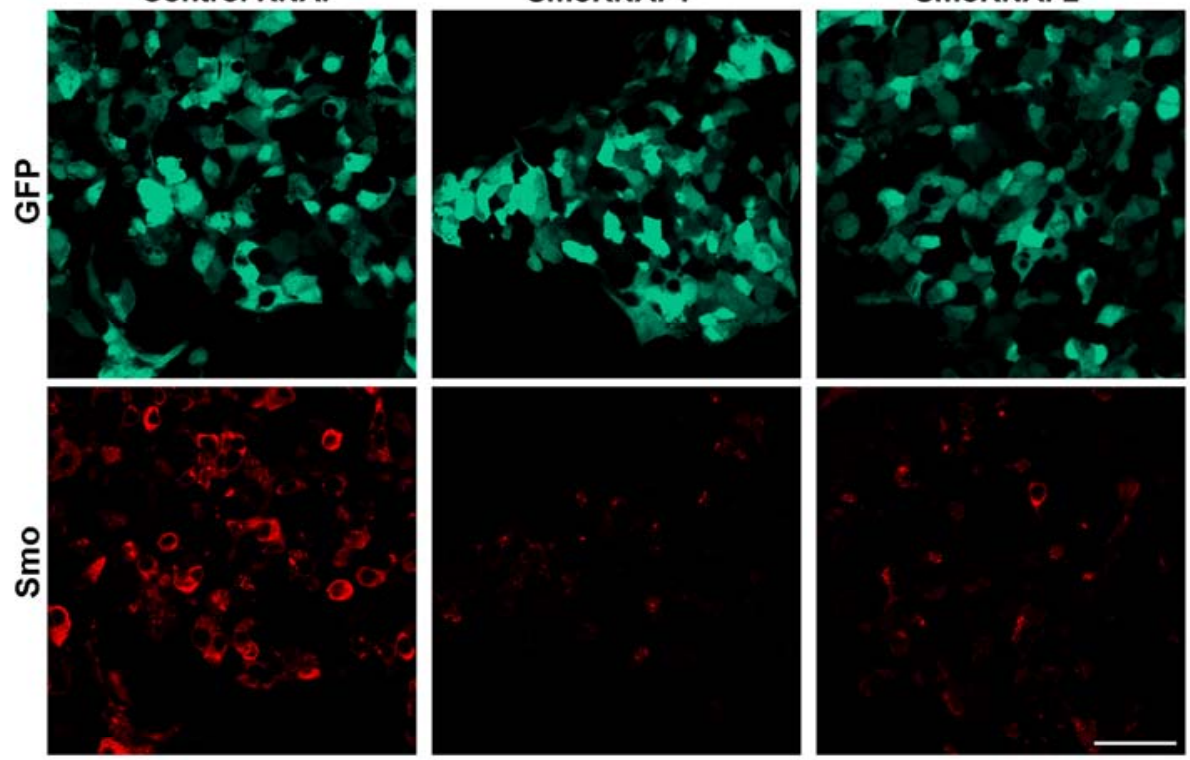

B

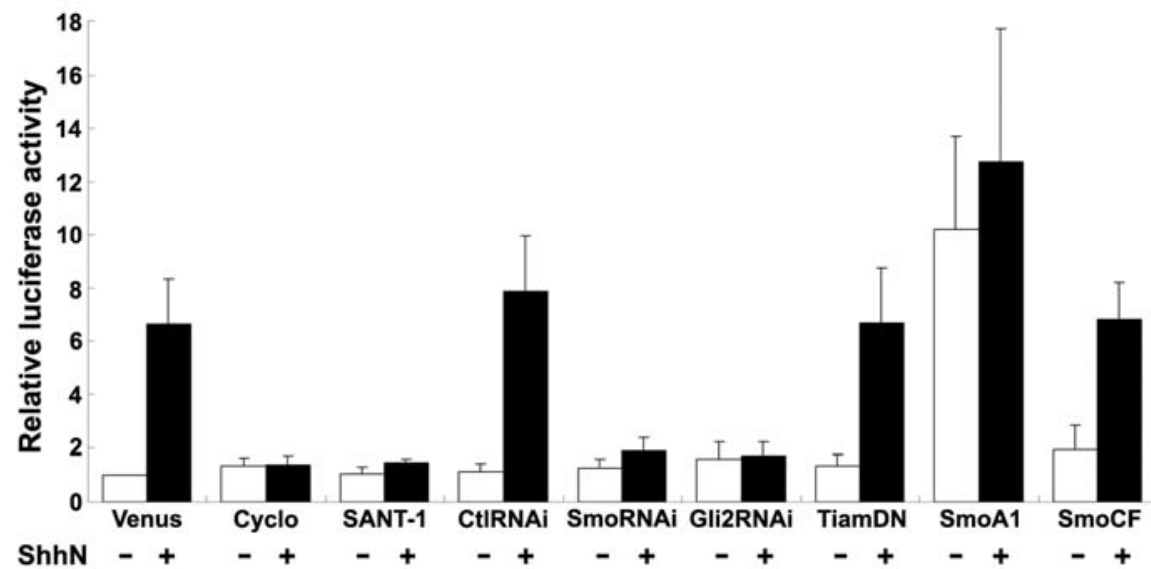

C
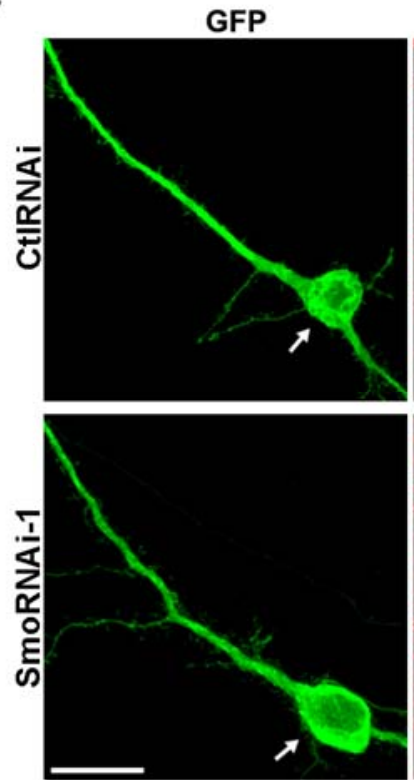

Smo
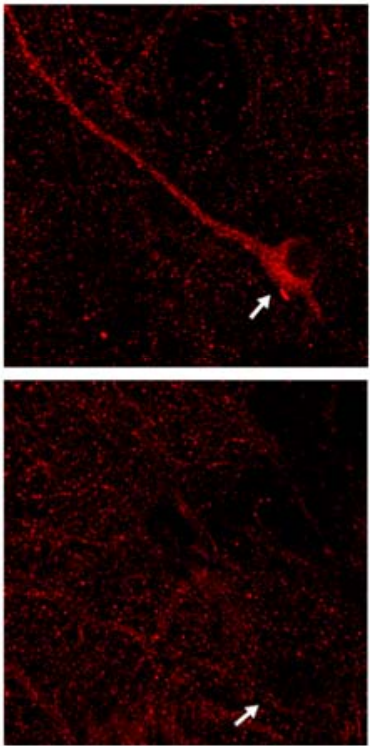

D

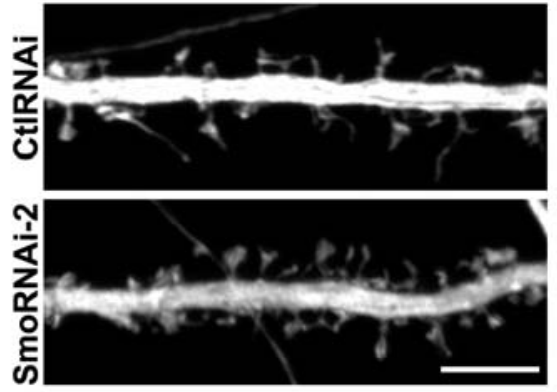




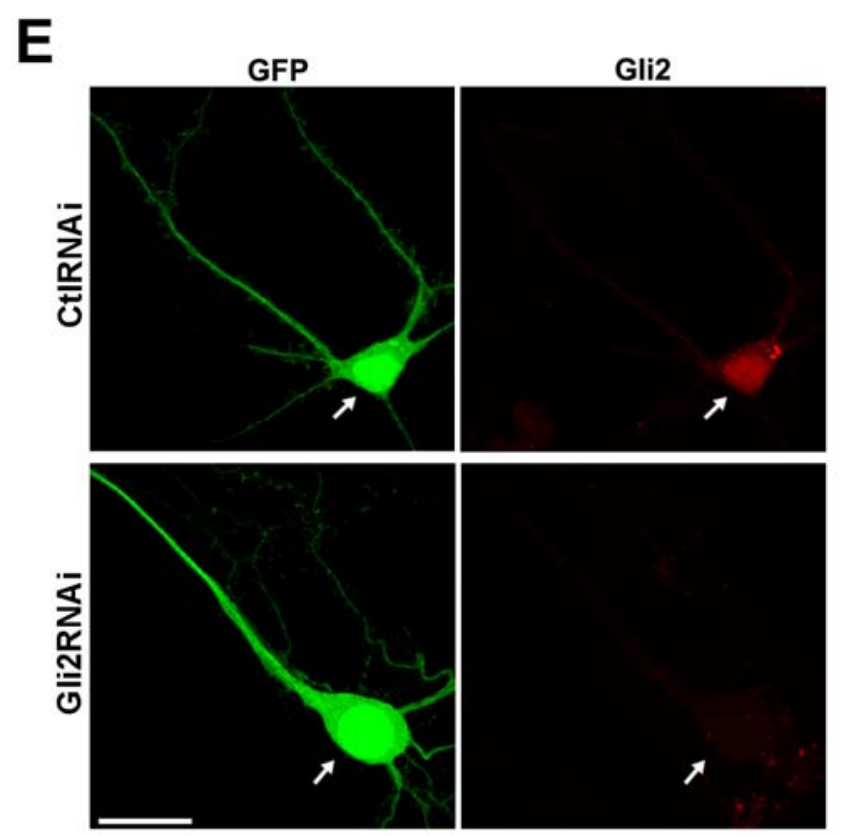

\section{Fig. S2. Effects of miR RNAi on Shh signaling}

(A) 293 cells were transfected with HA-tagged Smo together with negative control miR RNAi or Smo miR RNAi vectors. Cells were fixed and stained with an anti-HA antibody to verify the expression of HA-tagged Smo in GFP positive cells. Smo expression was inhibited by both SmoRNAi-1 and SmoRNAi-2. Scale bar represents $50 \mu \mathrm{m}$. (B) NIH 3 T3 cells were transiently transfected with the indicated constructs together with reporter constructs of Gli-luciferase and control Renilla luciferase. Luciferase activities were measured $72 \mathrm{hr}$ after treatment with Shh with or without cyclopamine or SANT-1 and normalized to values relative to control. (C) Hippocampal neurons were transfected with HA-tagged Smo together with the negative control RNAi or Smo RNAi-1 vectors. Cells were fixed and stained with an anti-HA antibody to verify the expression of recombinant Smo in GFP positive cells (arrows). Smo expression was inhibited by Smo RNAi-1. Scale bar represents $20 \mu \mathrm{m}$. (D) SmoRNAi-2 also induced spine formation in hippocampal neurons. 
Hippocampal neurons were transfected with SmoRNAi-2 and observed at 16 DIV. Scale bar represents $5 \mu \mathrm{m}$. (E) Hippocampal neurons were transfected with mCherry-tagged Gli2 together with the negative control RNAi or Gli2 RNAi vectors. Cells were fixed and the expression of recombinant Gli2 in GFP positive cells (arrows) was verified. Gli2 expression was inhibited by Gli2 RNAi. Scale bar represents $20 \mu \mathrm{m}$. 
Table S1. Transcriptional activation and spine formation by activation and inhibition of Shh pathway

\begin{tabular}{|c|c|c|c|c|c|c|c|}
\hline & Shh & SmoRNAi & SANT-1 & Gli2RNAi & SmoA1 & SmoCF & Cyclopamine \\
\hline $\begin{array}{c}\text { Transcriptional } \\
\text { activity }\end{array}$ & $\uparrow$ & $\downarrow$ & $\downarrow$ & $\downarrow$ & $\uparrow$ & $\rightarrow$ & $\downarrow$ \\
\hline $\begin{array}{c}\text { Spine } \\
\text { formation }\end{array}$ & $\uparrow$ & $\uparrow$ & $\uparrow$ & $\rightarrow$ & $\rightarrow$ & $\downarrow$ & $\downarrow$ \\
\hline
\end{tabular}

Transcriptional activity and spine formation are increased ( $\uparrow)$, unchanged $(\rightarrow)$ or decreased $(\downarrow)$ by indicated treatment. 